\title{
Shimura's mass formula for an orthogonal group over real quadratic fields
}

\author{
by \\ Manabu Murata (Shiga)
}

Introduction. Let $V$ be an $n$-dimensional row vector space over a totally real algebraic number field $F$, and let $\varphi$ be a totally positive definite symmetric matrix with entries in $F$. Put $\varphi[x]=x \varphi \cdot{ }^{t} x$ for $x \in V$. By a $\mathfrak{g}$ maximal lattice $L$ with respect to $\varphi$, we understand a $\mathfrak{g}$-lattice $L$ in $V$ which is maximal among $\mathfrak{g}$-lattices on which the values $\varphi[x]$ are contained in $\mathfrak{g}$. Here $\mathfrak{g}$ is the ring of integers of $F$. Put $G^{\varphi}=\left\{\alpha \in \mathrm{GL}_{n}(F) \mid \alpha \varphi \cdot{ }^{t} \alpha=\varphi\right\}$ and let $\left\{L_{i}\right\}_{i=1}^{k}$ be representatives of classes in the genus of $L$ with respect to $G^{\varphi}$. Then we define the mass of the genus of a $\mathfrak{g}$-maximal lattice $L$ with respect to $\varphi$ by

$$
\mathfrak{m}(L)=\sum_{i=1}^{k}\left[\Gamma_{i}: 1\right]^{-1},
$$

where $\Gamma_{i}=\left\{\gamma \in G^{\varphi} \mid L_{i} \gamma=L_{i}\right\}$. Shimura's mass formula for orthogonal groups determines $\mathfrak{m}(L)$ for an arbitrary totally real algebraic number field $F$ and arbitrary $\varphi$ ([6, Theorem 5.8]).

In this paper, we consider only the case where $F$ is a real quadratic field $\mathbb{Q}(\sqrt{m})$, and $\varphi$ is the unit matrix $1_{n}$ of size $n(n>1)$. The purpose of this paper is to digest Shimura's mass formula, applying it to the case of real quadratic fields, and to state the formula in a simpler form, from which we can compute $\mathfrak{m}(L)$. Applying this formula to the case where $F=\mathbb{Q}(\sqrt{5})$ and $\varphi=1_{4}$, we see that the genus belonging to a $\mathfrak{g}$-maximal lattice $L$ with respect to $\varphi$ consists of one class, and further give the number $N(L, h)=\#\{x \in L \mid$ $\varphi[x]=h\}$ for a totally positive element $h$ in $\mathfrak{g}$ by specializing the formula due to Shimura [7, Theorem 1.5] to the present situation (Section 4). At the end of this paper, we give a numerical table of $\mathfrak{m}(L)$ for several quadratic fields $F$ (Section 5).

Applying the formula in [6, Theorem 5.8] to our case, we can reduce the calculation of the mass to the following two arguments. One is to compute the special values of the Dedekind zeta function of $F$ and the $L$-function

2000 Mathematics Subject Classification: Primary 11E12; Secondary 11E20. 
of $F$ with the Hecke character of $F$ corresponding to $F(\sqrt{-1}) / F$ (Section 2). These values can be obtained by calculating the values of the Riemann zeta function and Dirichlet $L$-functions, since $F(\sqrt{-1}) / \mathbb{Q}$ is an abelian extension. The other is to determine a Witt decomposition for $1_{n}$ over the local field $F_{v}$ at a nonarchimedean prime $v$ of $F$ (Section 3). To do this, we first take an anisotropic part $\theta_{p}$ of a Witt decomposition for $1_{n}$ over $\mathbb{Q}_{p}$ for the rational prime $v \mid p$. Then we decompose $\theta_{p}$ on $F_{v}$. In particular, nontrivial cases are $v \mid 2$ and $n \equiv \pm 3,4(\bmod 8)$. In these cases, we can determine a Witt decomposition for $\theta_{p}$ over $F_{v}$ by using the local theory of quaternion algebras. Summing up these results, we obtain the mass $\mathfrak{m}(L)$ of the orthogonal group $G^{\varphi}$ of $\varphi=1_{n}$ over real quadratic fields $F$ (Theorem 3.6).

We end this introduction with the following remark: As was mentioned in [6], maximal lattices were introduced by Eichler. This maximal lattice differs from a unimodular lattice in general. Shimura gave the mass formula for the case of maximal lattices with respect to an arbitrary $\varphi$ exactly in [6, Theorem 5.8]. We use this formula to compute the mass in our case. Clearcut explanation why we work on maximal lattices is given in the introduction of [6] and also in that of [7].

Finally, I would like to thank my advisor Professor Koji Doi for suggesting this work and for guiding it to completion. I wish to thank Dr. Kaoru Okada for much help during the preparation of this paper. I also thank Dr. Yoshio Hiraoka for supporting our calculations in the numerical table.

Notation. If $R$ is an associative ring with identity element and if $M$ is an $R$-module, then we write $R^{\times}$for the group of all invertible elements of $R$, and $M_{n}^{m}$ for the $R$-module of $m \times n$-matrices with entries in $M$. We write $1_{n}$ for the identity element of the matrix ring $R_{n}^{n}$. We put $\mathrm{GL}_{n}(R)=\left(R_{n}^{n}\right)^{\times}$. We denote by ${ }^{t} x$ and $\operatorname{det}(x)$ the transpose and determinant of a matrix $x \in R_{n}^{n}$. If $x_{1}, \ldots, x_{r}$ are square matrices, $\operatorname{diag}\left[x_{1}, \ldots, x_{r}\right]$ denotes the matrix with $x_{1}, \ldots, x_{r}$ in the diagonal blocks and 0 in all other blocks. For a finite set $X$, we denote by $\# X$ the number of elements in $X$. For a symmetric matrix $A \in R_{n}^{n}$, we put

$$
A(x, y)=x A \cdot{ }^{t} y \quad \text { and } \quad A[x]=x A \cdot{ }^{t} x \quad\left(x, y \in R_{n}^{1}\right) .
$$

Let $F$ be a number field. We denote by $\mathbf{a}$ and $\mathbf{h}$ the sets of archimedean primes and nonarchimedean primes of $F$. We identify $v$ with the prime ideal of $F$ corresponding to $v$. For $v \in \mathbf{h}, \pi_{v}$ and $q_{v}$ denote a prime element of $F_{v}$ and the norm of the prime ideal at $v$. We denote by $B_{l}$ and $B_{l, \psi}$ the $l$ th Bernoulli number and $l$ th generalized Bernoulli number associated with a Dirichlet character $\psi$. For a rational prime $p,(\bar{p})$ denotes the Legendre symbol. 
1. Preliminaries. Throughout the paper, $F$ is a real quadratic field and $\varphi=1_{n}$. Let $\mathfrak{g}$ be the ring of integers of $F$ and put $V=F_{n}^{1}$. We put

$$
G=G^{\varphi}=\left\{\gamma \in \mathrm{GL}_{n}(F) \mid \gamma \varphi \cdot{ }^{t} \gamma=\varphi\right\}=\left\{\gamma \in \mathrm{GL}_{n}(F) \mid \gamma \cdot{ }^{t} \gamma=1_{n}\right\} .
$$

For a $\mathfrak{g}$-lattice $L$ in $V$, which is a finitely generated $\mathfrak{g}$-submodule in $V$ containing a basis of $V$, and $h \in \mathfrak{g}$, we put

$$
\begin{aligned}
N(L, h) & =\#\{x \in L \mid \varphi[x]=h\}, \\
\widetilde{L} & =\{y \in V \mid 2 \varphi(x, y) \in \mathfrak{g} \text { for every } x \in L\} .
\end{aligned}
$$

Let $G_{\mathbf{A}}$ be the adelization of $G$.

For $\alpha \in G_{\mathbf{A}}$, we denote by $L \alpha$ the $\mathfrak{g}$-lattice in $V$ such that $(L \alpha)_{v}=L_{v} \alpha_{v}$ for any nonarchimedean prime $v$ of $F$. Here $L_{v}$ is the $\mathfrak{g}_{v}$-lattice in $\left(F_{v}\right)_{n}^{1}$ which is spanned by $L$ over $\mathfrak{g}_{v}$. We call $\left\{L \alpha \mid \alpha \in G_{\mathbf{A}}\right\}$ (resp. $\{L \alpha \mid \alpha \in G\}$ ) the genus (resp. class) of $L$ with respect to $G$. We put

$$
C=\left\{\alpha \in G_{\mathbf{A}} \mid L \alpha=L\right\}, \quad \Gamma^{\alpha}=G \cap \alpha C \alpha^{-1} \quad\left(\alpha \in G_{\mathbf{A}}\right) .
$$

The mapping $G \alpha C \mapsto L \alpha^{-1}$ gives a bijection of $G \backslash G_{\mathbf{A}} / C$ onto the set of classes in the genus of $L$. It is known that $G \backslash G_{\mathbf{A}} / C$ is a finite set ([5, Lemma 8.7(4)]). Thus the genus of $L$ is decomposed into a disjoint union of finitely many classes. Let $\mathfrak{B}$ be a complete set of representatives for $G \backslash G_{\mathbf{A}} / C$. We then put

$$
\begin{aligned}
\mathfrak{m}(L) & =\mathfrak{m}(G, C)=\sum_{\alpha \in \mathfrak{B}}\left[\Gamma^{\alpha}: 1\right]^{-1}, \\
R(L, h) & =\sum_{\alpha \in \mathfrak{B}}\left[\Gamma^{\alpha}: 1\right]^{-1} N\left(L \alpha^{-1}, h\right) .
\end{aligned}
$$

We call $\mathfrak{m}(G, C)$ the mass of $G$ relative to $C$.

By a $\mathfrak{g}$-maximal lattice $L$ with respect to $\varphi$, we understand a $\mathfrak{g}$-lattice $L$ in $V$ which is maximal among $\mathfrak{g}$-lattices on which the values $\varphi[x]$ are contained in $\mathfrak{g}$. The genus of a $\mathfrak{g}$-maximal lattice $L$ consists of all $\mathfrak{g}$-maximal lattices (cf. [5, Lemma 5.9]).

Let $v \in \mathbf{a} \cup \mathbf{h}$. For symmetric matrices $A$ and $B$ in $\operatorname{GL}_{n}\left(F_{v}\right)$, we say that $A$ is equivalent to $B$ over $F_{v}$ if there exists $X \in \mathrm{GL}_{n}\left(F_{v}\right)$ such that $X A \cdot{ }^{t} X=B$. We call $A$ isotropic over $F_{v}$ if there exists $0 \neq x \in\left(F_{v}\right)_{n}^{1}$ such that $x A \cdot{ }^{t} x=0$. Otherwise we say that $A$ is anisotropic over $F_{v}$. Put

$$
\eta_{r}=\left[\begin{array}{cc}
0 & 1_{r} \\
1_{r} & 0
\end{array}\right]
$$

Then $\eta_{r}$ is a symmetric matrix of $\mathrm{GL}_{2 r}\left(F_{v}\right)$. For every $x \in\left(F_{v}\right)_{r}^{1}$, we have $\eta_{r}\left[\left[\begin{array}{ll}x & 0_{r}^{1}\end{array}\right]\right]=0$, and so $\eta_{r}$ is isotropic over $F_{v}$. 
For $\varphi$ and $v \in \mathbf{a} \cup \mathbf{h}$ there exists $\alpha_{v} \in \mathrm{GL}_{n}\left(F_{v}\right)$ such that

$$
\alpha_{v} \varphi \cdot{ }^{t} \alpha_{v}=\left[\begin{array}{ccc}
0 & 0 & 1_{r_{v}} \\
0 & \theta_{v} & 0 \\
1_{r_{v}} & 0 & 0
\end{array}\right]
$$

with an anisotropic symmetric matrix $\theta_{v} \in \mathrm{GL}_{t_{v}}\left(F_{v}\right)$ over $F_{v}$. We call (1.1) a Witt decomposition for $\varphi$ over $F_{v}$. Then $n=2 r_{v}+t_{v}$. It is known that $t_{v} \leq 4$ for $v \in \mathbf{h}$ (cf. [5, Proposition 5.2]). In Section 3, we determine a Witt decomposition for $\varphi$ over $F_{v}(v \in \mathbf{h})$.

2. Mass formula for $\varphi=1_{n}$ over real quadratic fields. Let $L$ be a $\mathfrak{g}$-maximal lattice in $V$ with respect to $\varphi$. The formula of $\mathfrak{m}(L)$ for $G$ is given in [6, Theorem 5.8]. In fact, we can apply this formula to the case of the maximal lattices with respect to an arbitrary totally definite quadratic form over an arbitrary totally real algebraic number field.

We first define a constant $\lambda_{v}$ in $[6$, Theorem 5.8]. Let $\mathfrak{e}$ be the product of all nonarchimedean primes $v$ satisfying $\widetilde{L}_{v} \neq L_{v}$. For $v \mid \mathfrak{e}$, put

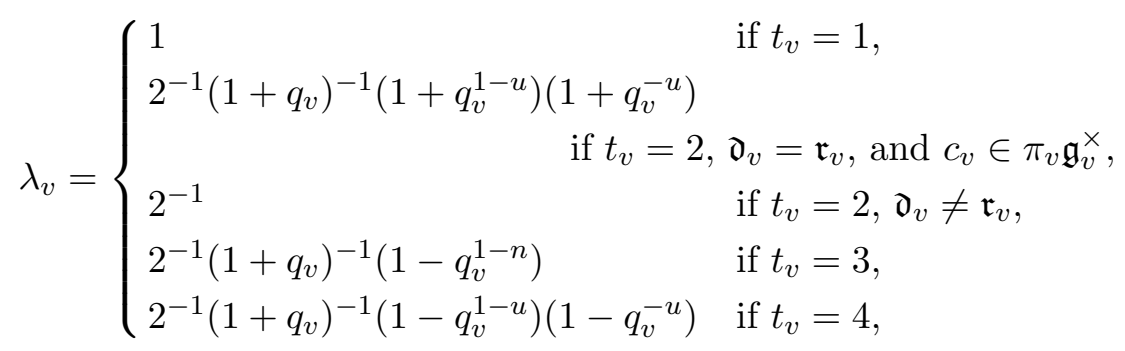

where $u=2^{-1} n$, and if $t_{v}=2$, then $\mathfrak{r}_{v}$ is the maximal order of $K_{v}=$ $F_{v}\left(\sqrt{-\operatorname{det}\left(\theta_{v}\right)}\right), \mathfrak{d}_{v}$ is the different of $K_{v}$ relative to $F_{v}$, and $c_{v}$ is a constant from $[6, \S 3.2]$. Here $c_{v}$ can be taken as follows: Let $\theta_{v}$ be as in (1.1). This is equivalent to a matrix $\operatorname{diag}\left[a_{v}, b_{v}\right]$ over $F_{v}$ with a suitable basis $\left\{e_{i}\right\}$ such that $a_{v}=\theta_{v}\left[e_{1}\right], b_{v}=\theta_{v}\left[e_{2}\right] \in F_{v}^{\times}$and $\theta_{v}\left(e_{1}, e_{2}\right)=0$. We may assume that $b_{v} \in \mathfrak{g}_{v}^{\times} \cup \pi_{v}^{-1} \mathfrak{g}_{v}^{\times}$. The mapping $y_{1} e_{1}+y_{2} e_{2} \mapsto b_{v} y_{2}+y_{1} \sqrt{-a_{v} b_{v}}$ yields an isomorphism $\left(F_{v}\right)_{2}^{1} \cong K_{v}$ such that

$$
\theta_{v}\left[y_{1} e_{1}+y_{2} e_{2}\right]=b_{v}^{-1} \cdot N_{K_{v} / F_{v}}\left(b_{v} y_{2}+y_{1} \sqrt{-a_{v} b_{v}}\right) .
$$

Here $N_{K_{v} / F_{v}}(x)=x x^{\varrho}$, where $\varrho$ is the nontrivial automorphism of $K_{v}$ over $F_{v}$. Then we can take $c_{v}=b_{v}^{-1}$. Notice that $v \nmid \mathfrak{e}$ if $t_{v}=0$, or $t_{v}=2$, $\mathfrak{d}_{v}=\mathfrak{r}_{v}$ and $c_{v} \in \mathfrak{g}_{v}^{\times}$(see (3.6) below).

Applying [6, Theorem 5.8] to the case where $F$ is a real quadratic field and $\varphi=1_{n}$, we have the following

Proposition 2.1. Let $F=\mathbb{Q}(\sqrt{m})$ with a square free positive integer $m$, and let $n>1$. Put $V=F_{n}^{1}$ and $\varphi=1_{n}$. Let $L$ be a $\mathfrak{g}$-maximal lattice in $V$ 
with respect to $\varphi, \mathfrak{e}$ the product of all nonarchimedean primes $v$ satisfying $\widetilde{L}_{v} \neq L_{v}$, and $\chi$ the Dirichlet character corresponding to $F / \mathbb{Q}$.

(1) Suppose $n$ is odd; let $\mathfrak{f}$ be the product of all nonarchimedean primes $v$ satisfying $t_{v}=3$. Then

$$
\mathfrak{m}(L)=2^{1-n}\left(\prod_{k=1}^{(n-1) / 2}(4 k)^{-2} B_{2 k} B_{2 k, \chi}\right)[\widetilde{L}: L]^{(n-1) / 2} \prod_{v \mid \mathfrak{f}} \frac{1-q_{v}^{1-n}}{2\left(1+q_{v}\right)} .
$$

(2) Suppose $n$ is even; put $F^{\prime}=\mathbb{Q}(\sqrt{-1})$ and $F^{\prime \prime}=\mathbb{Q}(\sqrt{-m})$, and denote by $\chi^{\prime}\left(\right.$ resp. $\left.\chi^{\prime \prime}\right)$ the Dirichlet character corresponding to $F^{\prime} / \mathbb{Q}$ $\left(\right.$ resp. $\left.F^{\prime \prime} / \mathbb{Q}\right)$. Let $\lambda_{v}$ be as in $(2.1)$.

(i) If $n \equiv 0(\bmod 4)$, then

$$
\begin{aligned}
\mathfrak{m}(L)= & n^{-2} B_{n / 2} B_{n / 2, \chi}\left(\prod_{k=1}^{[(n-1) / 2]}(4 k)^{-2} B_{2 k} B_{2 k, \chi}\right) \\
& \times[\widetilde{L}: L]^{(n-1) / 2} \prod_{v \mid \mathfrak{e}} \lambda_{v} .
\end{aligned}
$$

(ii) If $n \equiv 2(\bmod 4)$, then

$$
\begin{aligned}
\mathfrak{m}(L)= & n^{-2} B_{n / 2, \chi^{\prime}} B_{n / 2, \chi^{\prime \prime}} \\
& \times\left(\prod_{k=1}^{[(n-1) / 2]}(4 k)^{-2} B_{2 k} B_{2 k, \chi}\right)[\widetilde{L}: L]^{(n-1) / 2} \\
& \times \prod_{v \mid \mathfrak{e}} \lambda_{v} \cdot \begin{cases}2^{2(1-n)} & \text { if } m \equiv 1(\bmod 4), \\
2^{1-n} & \text { if } m \equiv 2(\bmod 4), \\
1 & \text { if } m \equiv 3(\bmod 4) .\end{cases}
\end{aligned}
$$

Proof. By [6, Lemma 5.6(1)], we have

$$
\mathfrak{m}(G, C)=\frac{1}{2} \mathfrak{m}\left(G_{+}, C^{+}\right),
$$

where

$$
G_{+}=\{\gamma \in G \mid \operatorname{det}(\gamma)=1\}
$$

and $C^{+}=C \cap\left(G_{+}\right)_{\mathbf{A}}$ with the adelization $\left(G_{+}\right)_{\mathbf{A}}$ of $G_{+}$. Taking $\mathfrak{g}$ as an ideal $\mathfrak{c}$ of $F$ in [6, Theorem 5.8], we have $C^{+}=D^{+}$, where $D^{+}$is a subgroup of $\left(G_{+}\right)_{\mathbf{A}}$ in that theorem (cf. [6, (5.7.3), (5.7.4), and (5.7.5)]). Since $\varphi$ is anisotropic over $F$ and satisfies the condition [6, (5.7.1)], we can apply [6, Theorem 5.8] to $\mathfrak{m}\left(G_{+}, C^{+}\right)$. Hence we obtain

$$
\mathfrak{m}\left(G_{+}, C^{+}\right)=\mathfrak{m}_{n}(\mathfrak{g})[\widetilde{L}: L]^{\mu} \prod_{v \mid \mathfrak{e}} \lambda_{v},
$$


where

$$
\begin{aligned}
\mathfrak{m}_{n}(\mathfrak{g})= & 2 D_{F}^{\left[\mu^{2}\right]} \prod_{k=1}^{[\mu]}\left\{D_{F}^{1 / 2}\left((2 k-1) !(2 \pi)^{-2 k}\right)^{2} \zeta_{F}(2 k)\right\} \\
& \times \begin{cases}2^{-2 \mu} & \text { if } n \text { is odd } \\
D_{F}^{1 / 2}\left((u-1) !(2 \pi)^{-u}\right)^{2} L\left(u, \psi_{K / F}\right) & \text { if } n \text { is even. }\end{cases}
\end{aligned}
$$

Here $\mu=(n-1) / 2$, and $D_{F}$ is the discriminant of $F$; if $n$ is even, put $u=n / 2, K=F\left(\sqrt{(-1)^{u}}\right)$, and denote by $\psi_{K / F}$ the Hecke character of $F$ corresponding to $K / F$.

Suppose $n$ is odd. Then we have $D_{F}^{\left[\mu^{2}\right]}=\prod_{k=1}^{[\mu]} D_{F}^{2 k-1}$, and hence

$$
\mathfrak{m}_{n}(\mathfrak{g})=2^{-(n-2)} \prod_{k=1}^{[\mu]} D_{F}^{2 k-1 / 2}\left((2 k-1) !(2 \pi)^{-2 k}\right)^{2} \zeta_{F}(2 k) .
$$

Put $\widehat{\zeta}_{F}(s)=\pi^{-s} \Gamma(s / 2)^{2} \zeta_{F}(s)$, where $\Gamma(s)$ is the gamma function $(s \in \mathbb{C})$. By the functional equation $\widehat{\zeta}_{F}(s)=\left|D_{F}\right|^{1 / 2-s} \widehat{\zeta}_{F}(1-s)$, we have

$$
\zeta_{F}(2 k)=\left|D_{F}\right|^{1 / 2-2 k} \pi^{4 k}\left(\frac{2^{2 k-1}}{(2 k-1) !}\right)^{2} \zeta_{F}(1-2 k) .
$$

Thus

$$
D_{F}^{2 k-1 / 2}\left((2 k-1) !(2 \pi)^{-2 k}\right)^{2} \zeta_{F}(2 k)=2^{-2} \zeta_{F}(1-2 k)
$$

Since

$$
\begin{aligned}
\zeta_{F}(s) & =\zeta_{\mathbb{Q}}(s) L(s, \chi), \\
\zeta_{\mathbb{Q}}(1-2 k) & =-B_{2 k} / 2 k, \\
L(1-2 k, \chi) & =-B_{2 k, \chi} / 2 k,
\end{aligned}
$$

we have

$$
D_{F}^{2 k-1 / 2}\left((2 k-1) !(2 \pi)^{-2 k}\right)^{2} \zeta_{F}(2 k)=(4 k)^{-2} B_{2 k} B_{2 k, \chi} .
$$

Substituting this into (2.5) gives

$$
\mathfrak{m}_{n}(\mathfrak{g})=2^{-(n-2)} \prod_{k=1}^{[\mu]}(4 k)^{-2} B_{2 k} B_{2 k, \chi}
$$

and hence we obtain (2.2).

Suppose $n$ is even. Since $D_{F}^{\left[\mu^{2}\right]}=D_{F}^{u-1} \prod_{k=1}^{[\mu]} D_{F}^{2 k-1}$, we have

$$
\mathfrak{m}_{n}(\mathfrak{g})=2 D_{F}^{\mu}\left((u-1) !(2 \pi)^{-u}\right)^{2} L\left(u, \psi_{K / F}\right) \prod_{k=1}^{[\mu]}(4 k)^{-2} B_{2 k} B_{2 k, \chi} .
$$


Then

$$
\begin{aligned}
\mathfrak{m}(L)= & D_{F}^{\mu}\left((u-1) !(2 \pi)^{-u}\right)^{2} L\left(u, \psi_{K / F}\right) \\
& \times\left(\prod_{k=1}^{[\mu]}(4 k)^{-2} B_{2 k} B_{2 k, \chi}\right)[\widetilde{L}: L]^{\mu} \prod_{v \mid \mathfrak{e}} \lambda_{v} .
\end{aligned}
$$

Next, we compute the value $L\left(u, \psi_{K / F}\right)$. If $n \equiv 0(\bmod 4)$, then $K=F$, and so $L\left(u, \psi_{K / F}\right)=\zeta_{F}(u)$. Since this value can be obtained as above, we easily deduce $(2.3)$ from $(2.6)$. Let $n \equiv 2(\bmod 4)$. Then $K$ is a totally imaginary quadratic field $F(\sqrt{-1})=\mathbb{Q}(\sqrt{m}, \sqrt{-1})$ over $F$. In this case, the functional equation is $R\left(s, \psi_{K / F}\right)=R\left(1-s, \psi_{K / F}\right)$ by [5, Theorems A6.2, A6.3], where

$$
R\left(s, \psi_{K / F}\right)=\left|D_{F} N\left(D_{K / F}\right)\right|^{s / 2}\left(\pi^{-(s+1) / 2} \Gamma((s+1) / 2)\right)^{2} L\left(s, \psi_{K / F}\right) .
$$

From this,

$$
L\left(s, \psi_{K / F}\right)=\left|D_{F} N\left(D_{K / F}\right)\right|^{(1-2 s) / 2} \pi^{2 s-1}\left(\frac{\Gamma((2-s) / 2)}{\Gamma((s+1) / 2)}\right)^{2} L\left(1-s, \psi_{K / F}\right) .
$$

Now, since $K / \mathbb{Q}, F / \mathbb{Q}$, and $K / F$ are abelian extensions, we have

$$
\begin{aligned}
& \zeta_{K}(s)=\zeta_{\mathbb{Q}}(s) L(s, \chi) L\left(s, \chi^{\prime}\right) L\left(s, \chi^{\prime \prime}\right), \\
& \zeta_{F}(s)=\zeta_{\mathbb{Q}}(s) L(s, \chi), \\
& \zeta_{K}(s)=\zeta_{F}(s) L\left(s, \psi_{K / F}\right) .
\end{aligned}
$$

Hence we obtain

$$
L\left(s, \psi_{K / F}\right)=\zeta_{K}(s) \zeta_{F}(s)^{-1}=L\left(s, \chi^{\prime}\right) L\left(s, \chi^{\prime \prime}\right) .
$$

It is known that $L(1-k, \omega)=-B_{k, \omega} / k$ for a positive odd integer $k$ with $\omega=\chi^{\prime}$ or $\chi^{\prime \prime}$. Combining this with $(2.7)$, we find that $L\left(1-u, \psi_{K / F}\right)=$ $u^{-2} B_{u, \chi^{\prime}} B_{u, \chi^{\prime \prime}}$. Observing

$$
\frac{\Gamma((2-u) / 2)}{\Gamma((u+1) / 2)}=\frac{(-1)^{(u-1) / 2} 2^{u-1} \pi^{1 / 2}}{(u-1) !},
$$

we see that

$$
D_{F}^{\mu}\left((u-1) !(2 \pi)^{-u}\right)^{2} L\left(u, \psi_{K / F}\right)=n^{-2} N\left(D_{K / F}\right)^{-\mu} B_{u, \chi^{\prime}} B_{u, \chi^{\prime \prime}} .
$$

It is known that $D_{K}=D_{F} D_{F^{\prime}} D_{F^{\prime \prime}}$. Since $D_{K}=N\left(D_{K / F}\right) D_{F}^{2}$, we have

$$
\begin{aligned}
N\left(D_{K / F}\right) & =D_{F}^{-1} D_{F^{\prime}} D_{F^{\prime \prime}}=4 D_{F}^{-1} D_{F^{\prime \prime}} \\
& = \begin{cases}2^{4} & \text { if } m \equiv 1(\bmod 4), \\
2^{2} & \text { if } m \equiv 2(\bmod 4), \\
1 & \text { if } m \equiv 3(\bmod 4) .\end{cases}
\end{aligned}
$$

Substituting these into (2.6), we obtain (2.4). 


\section{Computation of the mass}

3.1. Local Witt decompositions for $\varphi=1_{n}$. Throughout this section, $L$ is a $\mathfrak{g}$-maximal lattice in $V$ with respect to $\varphi$. To compute $\mathfrak{m}(L)$, we need to obtain $\mathfrak{e}, \mathfrak{f},[\widetilde{L}: L]$, and $\lambda_{v}$ of Proposition 2.1. First of all, we determine a Witt decomposition for $\varphi=1_{n}$ at each nonarchimedean prime $v$ of $F$ (Lemma 3.3). For this purpose, we use a quaternion algebra for $v \mid 2$ and $n \equiv \pm 3,4(\bmod 8)$. By Wedderburn's theorem, every quaternion algebra over $F_{v}$ is isomorphic to the matrix algebra $\left(F_{v}\right)_{2}^{2}$ or a division algebra. For a quaternion algebra $B$ over $F$ and $v \in \mathbf{a} \cup \mathbf{h}$, we say that $B$ is ramified at $v$ if $B \otimes_{F} F_{v}$ is a division algebra, and that $B$ is unramified at $v$ if $B \otimes_{F} F_{v}$ is isomorphic to $\left(F_{v}\right)_{2}^{2}$. It is well known that $B$ is ramified at $v$ if and only if the reduced norm of $B \otimes_{F} F_{v}$ is anisotropic over $F_{v}$, and that the reduced norm of the matrix algebra $\left(F_{v}\right)_{2}^{2}$ is equivalent to the isotropic symmetric matrix $\eta_{2}$.

Lemma 3.1. Let $p$ be a rational prime. Then we can take an anisotropic symmetric matrix $\theta_{p}$ of a Witt decomposition for $\varphi$ over $\mathbb{Q}_{p}$ of the following form:

(1) If $p \equiv 1(\bmod 4)$, then

$$
\theta_{p}= \begin{cases}\emptyset & \text { if } n \text { is even } \\ 1 & \text { if } n \text { is odd. }\end{cases}
$$

(2) If $p \equiv 3(\bmod 4)$, then

$$
\theta_{p}= \begin{cases}\emptyset & \text { if } n \equiv 0(\bmod 4) \\ \pm 1 & \text { if } n \equiv \pm 1(\bmod 4), \\ 1_{2} & \text { if } n \equiv 2(\bmod 4) .\end{cases}
$$

(3) If $p=2$, then

$$
\theta_{p}= \begin{cases}\emptyset & \text { if } n \equiv 0(\bmod 8) \\ \pm 1 & \text { if } n \equiv \pm 1(\bmod 8) \\ \pm 1_{2} & \text { if } n \equiv \pm 2(\bmod 8) \\ \pm 1_{3} & \text { if } n \equiv \pm 3(\bmod 8) \\ 1_{4} & \text { if } n \equiv 4(\bmod 8) .\end{cases}
$$

Here $\theta_{p}=\emptyset$ means that $t_{p}=0$, i.e. $\varphi$ is equivalent to $\eta_{n / 2}$ over $\mathbb{Q}_{p}$, and $" \theta_{p}= \pm 1$ if $n \equiv \pm 1(\bmod 4) "$ means that $\theta_{p}=1\left(\right.$ resp. $\left.\theta_{p}=-1\right)$ if $n \equiv 1$ $(\bmod 4)($ resp. $n \equiv-1(\bmod 4))$. The other double signs should be read similarly.

This fact can be seen by [6, Examples 5.16] or [8, (1.2)]. We remark that the constant $c_{p}$ in $[8,(1.2)]$ is determined by the determinant of the decomposition. 
We next give a Witt decomposition for $\varphi$ over $F_{v}$ for a totally real number field $F$, though we are interested in real quadratic ones. To do this, we define the Hilbert symbol $(a, b)_{F_{v}}$ over $F_{v}$ by

$$
(a, b)_{F_{v}}= \begin{cases}1 & \text { if } \operatorname{diag}[a, b,-1] \text { is isotropic over } F_{v}, \\ -1 & \text { otherwise }\end{cases}
$$

for $a, b \in F_{v}^{\times}$. We can verify that $(a, b)_{F_{v}}=1$ if and only if there exists $0 \neq[x y] \in\left(F_{v}\right)_{2}^{1}$ such that $a x^{2}+b y^{2}=1$.

Lemma 3.2. Let $F$ be a totally real number field. Let $v \in \mathbf{h}$, and let $p$ be the rational prime which lies below $v$. Then we can take an anisotropic symmetric matrix $\theta_{v}$ of a Witt decomposition for $\varphi$ over $F_{v}$ of the following form:

(1) Suppose $v \nmid 2$.

(i) If $p \equiv 1(\bmod 4)$, then

$$
\theta_{v}= \begin{cases}\emptyset & \text { if } n \text { is even } \\ 1 & \text { if } n \text { is odd }\end{cases}
$$

(ii) If $p \equiv 3(\bmod 4)$ and $\sqrt{-1} \notin F_{v}$, then

$$
\theta_{v}= \begin{cases}\emptyset & \text { if } n \equiv 0(\bmod 4), \\ \pm 1 & \text { if } n \equiv \pm 1(\bmod 4), \\ 1_{2} & \text { if } n \equiv 2(\bmod 4) .\end{cases}
$$

(iii) If $p \equiv 3(\bmod 4)$ and $\sqrt{-1} \in F_{v}$, then

$$
\theta_{v}= \begin{cases}\emptyset & \text { if } n \text { is even } \\ 1 & \text { if } n \text { is odd. }\end{cases}
$$

(2) Suppose $v \mid 2$.

(i) If $n \equiv 0(\bmod 8)$, then $\theta_{v}=\emptyset$.

(ii) If $n \equiv \pm 1(\bmod 8)$, then $\theta_{v}= \pm 1$.

(iii) If $n \equiv \pm 2(\bmod 8)$, then

$$
\theta_{v}= \begin{cases}\emptyset & \text { if } \sqrt{-1} \in F_{v} \\ \pm 1_{2} & \text { if } \sqrt{-1} \notin F_{v} .\end{cases}
$$

(iv) If $n \equiv \pm 3(\bmod 8)$, then

$$
\theta_{v}= \begin{cases} \pm 1_{3} & \text { if }(-1,-1)_{F_{v}}=-1 \\ \mp 1 & \text { if }(-1,-1)_{F_{v}}=1 .\end{cases}
$$

(v) If $n \equiv 4(\bmod 8)$, then

$$
\theta_{v}= \begin{cases}1_{4} & \text { if }(-1,-1)_{F_{v}}=-1 \\ \emptyset & \text { if }(-1,-1)_{F_{v}}=1\end{cases}
$$

Here $\theta_{v}=\emptyset$ means that $t_{v}=0$. 
Proof. (1) Suppose $v \nmid 2$. Let $\theta_{p}$ be as in Lemma 3.1. If $p \equiv 1(\bmod 4)$, then we can take $\theta_{v}=\theta_{p}$, and thus we have (i).

To prove (ii) and (iii), let $p \equiv 3(\bmod 4)$. We show only the case of $n \equiv 2$ $(\bmod 4)$, since the other cases are trivial. In this case,

$$
1_{2} \text { is isotropic over } F_{v} \Leftrightarrow \sqrt{-1} \in F_{v},
$$

which proves (ii) and (iii).

(2) Suppose $v \mid 2$. If $n \equiv 0, \pm 1$, or $\pm 2(\bmod 8)$, then we can determine a decomposition for $\varphi$ over $F_{v}$ in the same way as in (1), which proves (i)-(iii).

To prove (iv) and (v), we take the quaternion algebra $B_{0}$ of $[6$, Examples 5.16] which is only ramified at 2 and infinity: $B_{0}=\mathbb{Q}+\mathbb{Q} a+\mathbb{Q} b+\mathbb{Q} a b$, where $a^{2}=b^{2}=-1$ and $b a=-a b$. Then the reduced norm of $B_{0}$ is equivalent to $1_{4}$ over $\mathbb{Q}$. Put $B=B_{0} \otimes_{\mathbb{Q}} F$. Then it can be verified that

$$
\begin{aligned}
(-1,-1)_{F_{v}}=1 & \Leftrightarrow-1 \in N_{F_{v}(\sqrt{-1}) / F_{v}}\left(F_{v}(\sqrt{-1})^{\times}\right) \\
& \Leftrightarrow \text { the reduced norm } N_{B / F} \text { of } B \text { is isotropic over } F_{v},
\end{aligned}
$$

which leads to (v). Furthermore, set $W=\left\{x \in B \mid \operatorname{Tr}_{B / F}(x)=0\right\}$ and let $\psi$ be the restriction of $N_{B / F}$ to $W$. Then $\psi$ is equivalent to $1_{3}$ over $F$, and it can be verified that $N_{B / F}$ is isotropic over $F_{v}$ if and only if $\psi$ is isotropic over $F_{v}$, which proves (iv).

Returning to real quadratic fields, we have

Lemma 3.3. Let $F=\mathbb{Q}(\sqrt{m})$ with a square free positive integer $m$. Let $v \in \mathbf{h}$, and let $p$ be the rational prime which lies below $v$. Then we can take an anisotropic symmetric matrix $\theta_{v}$ of a Witt decomposition for $\varphi$ over $F_{v}$ of the following form:

(1) Suppose $v \nmid 2$.

(i) If $p \equiv 1(\bmod 4)$, then

$$
\theta_{v}= \begin{cases}\emptyset & \text { if } n \text { is even } \\ 1 & \text { if } n \text { is odd }\end{cases}
$$

(ii) If $p \equiv 3(\bmod 4)$ and $\left(\frac{D_{F}}{p}\right) \neq-1$, then

$$
\theta_{v}= \begin{cases}\emptyset & \text { if } n \equiv 0(\bmod 4), \\ \pm 1 & \text { if } n \equiv \pm 1(\bmod 4), \\ 1_{2} & \text { if } n \equiv 2(\bmod 4) .\end{cases}
$$

(iii) If $p \equiv 3(\bmod 4)$ and $\left(\frac{D_{F}}{p}\right)=-1$, then

$$
\theta_{v}= \begin{cases}\emptyset & \text { if } n \text { is even } \\ 1 & \text { if } n \text { is odd. }\end{cases}
$$


(2) Suppose $v \mid 2$.

(i) If $n \equiv 0(\bmod 8)$, then $\theta_{v}=\emptyset$.

(ii) If $n \equiv \pm 1(\bmod 8)$, then $\theta_{v}= \pm 1$.

(iii) If $n \equiv \pm 2(\bmod 8)$, then

$$
\theta_{v}= \begin{cases}\emptyset & \text { if } m \equiv-1(\bmod 8) \\ \pm 1_{2} & \text { if } m \not \equiv-1(\bmod 8) .\end{cases}
$$

(iv) If $n \equiv \pm 3(\bmod 8)$, then

$$
\theta_{v}= \begin{cases} \pm 1_{3} & \text { if } m \equiv 1(\bmod 8) \\ \mp 1 & \text { if } m \neq \equiv 1(\bmod 8) .\end{cases}
$$

(v) If $n \equiv 4(\bmod 8)$, then

$$
\theta_{v}= \begin{cases}1_{4} & \text { if } m \equiv 1(\bmod 8) \\ \emptyset & \text { if } m \neq \equiv 1(\bmod 8) .\end{cases}
$$

Here $\theta_{v}=\emptyset$ means that $t_{v}=0$.

Proof. First, we note that $\sqrt{d} \in \mathbb{Q}_{p}$ if and only if $p$ splits in the quadratic field $\mathbb{Q}(\sqrt{d})$. We see that if $p \not \equiv 1(\bmod 4)$, that is, $\sqrt{-1} \notin \mathbb{Q}_{p}$, then

$$
\sqrt{-1} \in F_{v} \Leftrightarrow \sqrt{-m} \in \mathbb{Q}_{p} .
$$

To show this equivalence, we may assume that $\left[F_{v}: \mathbb{Q}_{p}\right]=2$. Let $\sqrt{-1} \in F_{v}$. As $\sqrt{m} \in F_{v}$, there exist $x, y \in \mathbb{Q}_{p}$ such that $\sqrt{-m}=x+y \sqrt{m}$. Thus $-m=x^{2}+m y^{2}+2 x y \sqrt{m}$. If $x=0$, then $\sqrt{-1} \in \mathbb{Q}_{p}$. This contradicts $\sqrt{-1} \notin \mathbb{Q}_{p}$. Hence $y=0$ and $x^{2}=-m$. The converse is trivial.

(1) Suppose $v \nmid 2$. Let $\theta_{p}$ be as in Lemma 3.1. If $p \equiv 1(\bmod 4)$, then we can take $\theta_{v}=\theta_{p}$, and thus we have (i).

To prove (ii) and (iii), let $p \equiv 3(\bmod 4)$. We show only the case of $n \equiv 2$ $(\bmod 4)$, since the other cases are trivial. In this case, $\theta_{p}=1_{2}$. If $p$ splits in $F$, then $\sqrt{-1} \notin \mathbb{Q}_{p}=F_{v}$. If $p$ does not split in $F$, then

$$
p \text { remains prime in } F \Leftrightarrow\left(\frac{-m}{p}\right)=-\left(\frac{D_{F}}{p}\right)=1 \Leftrightarrow \sqrt{-m} \in \mathbb{Q}_{p},
$$

since $D_{F}=m$ or $4 m,\left(\frac{2}{p}\right) \neq 0$, and $\left(\frac{-1}{p}\right)=-1$. Thus, by (3.3) and (3.2), if $p$ splits or ramifies in $F$, then $1_{2}$ is anisotropic over $F_{v}$. If $p$ remains prime in $F$, then $1_{2}$ is isotropic, and equivalent to $\eta_{1}$ over $F_{v}$ by

$$
\alpha 1_{2} \cdot{ }^{t} \alpha=\eta_{1} \quad \text { with } \quad \alpha=\left[\begin{array}{cc}
1 & \sqrt{-1} \\
1 / 2 & -\sqrt{-1} / 2
\end{array}\right] .
$$

(2) Suppose $v \mid 2$. We note that $\sqrt{-1} \notin \mathbb{Q}_{2}$. By (3.3) and (3.2), we know that $m \equiv-1(\bmod 8)$ if and only if $\sqrt{-1} \in F_{v}$. In this case, using the same method as in case (1)(iii), we can find a decomposition for $\varphi$ over $F_{v}$, since 1 is equivalent to -1 over $F_{v}$. 
We assume $m \not \equiv-1(\bmod 8)$ until the end of this proof. If $n \equiv 0, \pm 1$, or $\pm 2(\bmod 8)$, then we can take the decomposition for $\varphi$ over $F_{v}$ as in Lemma 3.2 because of (3.3), which proves (i)-(iii) for $m \not \equiv-1(\bmod 8)$.

To prove (v), though we have given a decomposition for $1_{4}$ by using the Hilbert symbol in Lemma 3.2, we now determine it in another way. We again take $B_{0}$ and $B$ as in Lemma 3.2. Since $B_{0} \otimes_{\mathbb{Q}} \mathbb{Q}_{2}$ is a division algebra, $1_{4}$ is anisotropic over $\mathbb{Q}_{2}$. By the local theory of quaternion algebras over algebraic number fields, a quaternion algebra over a nonarchimedean local field splits over an arbitrary quadratic field over the local field (cf. [1, VII, $\S 2$, Satz 4]). From this fact, we obtain

$$
\begin{aligned}
B \otimes_{F} F_{v} & \cong\left(B_{0} \otimes_{\mathbb{Q}} \mathbb{Q}_{2}\right) \otimes_{\mathbb{Q}_{2}} F_{v} \\
& \cong \begin{cases}B_{0} \otimes_{\mathbb{Q}} \mathbb{Q}_{2} & \text { if } 2 \text { splits in } F, \\
\left(F_{v}\right)_{2}^{2} & \text { if } 2 \text { ramifies or remains prime in } F .\end{cases}
\end{aligned}
$$

Therefore we have

$$
\begin{cases}1_{4} \text { is anisotropic over } F_{v} & \text { if } m \equiv 1(\bmod 8), \\ 1_{4} \text { is equivalent to } \eta_{2} \text { over } F_{v} & \text { if } m \neq \equiv 1(\bmod 8) .\end{cases}
$$

Next, we prove the case $n \equiv 3(\bmod 8)$; the proof of the case $n \equiv-3$ $(\bmod 8)$ can be obtained in the same way. Then we have

$$
\begin{cases}1_{3} \text { is anisotropic over } F_{v} & \text { if } m \equiv 1(\bmod 8), \\ 1_{3} \text { is equivalent to } \operatorname{diag}\left[\eta_{1},-1\right] \text { over } F_{v} & \text { if } m \neq \equiv 1(\bmod 8) .\end{cases}
$$

To show this, we first assume that $m \equiv 1(\bmod 8)$. Since $1_{4}$ is anisotropic over $F_{v}$, so is $1_{3}$. Next assume $m \not \equiv 1(\bmod 8)$. Then there exists an element $\beta_{v}$ in $\mathrm{GL}_{4}\left(F_{v}\right)$ such that $\beta_{v} 1_{4} \cdot{ }^{t} \beta_{v}=\eta_{2}$. We identify $\left(F_{v}\right)_{3}^{1}$ with the subspace of $\left(F_{v}\right)_{4}^{1}$ by the mapping $\left[x_{1} x_{2} x_{3}\right] \mapsto\left[x_{1} x_{2} x_{3} 0\right]$. Put $H=\left(F_{v}\right)_{4}^{1} \beta_{v}^{-1}$ and $W=\left(F_{v}\right)_{3}^{1} \beta_{v}^{-1}$. Now $W \cap\left(F_{v}\right)_{2}^{1} \neq\{0\}$ in $H$. Hence there exists $0 \neq y \in\left(F_{v}\right)_{3}^{1}$ such that $y \beta_{v}^{-1} \in W \cap\left(F_{v}\right)_{2}^{1}$. Then $1_{3}[y]=1_{4}[y]=\eta_{2}\left[y \beta_{v}^{-1}\right]=0$, and so $1_{3}$ is isotropic over $F_{v}$. Put $y=\left[y_{1} y_{2} y_{3}\right]$. Then $y_{1}^{2}+y_{2}^{2}+y_{3}^{2}=0$. We may assume that $y_{2} \neq 0$. Then we have $N_{F_{v}(\sqrt{-1}) / F_{v}}(z)=-1$ with $z=y_{1} \sqrt{-1}\left(y_{2}+y_{3} \sqrt{-1}\right)^{-1} \in F_{v}(\sqrt{-1})$. From this, $1_{2}$ is equivalent to $-1_{2}$ over $F_{v}$. Thus $1_{3}=\operatorname{diag}\left[1,1_{2}\right]$ is equivalent to $\operatorname{diag}\left[\eta_{1},-1\right]$, which is the desired decomposition.

3.2. The relative discriminant $D_{K / F}$. Put $K=F(\sqrt{-1})$. We give the relative discriminant $D_{K / F}$ and the relative different $\mathfrak{d}_{K / F}$ of $K / F$, which is needed in the proof of Lemma 3.4:

$$
D_{K / F}= \begin{cases}4 \mathfrak{g} & \text { if } m \equiv 1(\bmod 4) \\ 2 \mathfrak{g} & \text { if } m \equiv 2(\bmod 4), \\ \mathfrak{g} & \text { if } m \equiv 3(\bmod 4),\end{cases}
$$




$$
\mathfrak{d}_{K / F}= \begin{cases}\prod_{v} v \mathfrak{r} & \text { if } m \equiv 1,2(\bmod 4) \\ \mathfrak{r} & \text { if } m \equiv 3(\bmod 4)\end{cases}
$$

where $\mathfrak{r}$ is the ring of integers of $K$, and the product runs through primes $v$ in $F$ dividing 2. To show these, we first determine $D_{K / F}$. We see that $v \mid D_{K / F}$ only if $v \mid 2$ by $(2.8)$. If $m \equiv 5(\bmod 8)$, then $D_{K / F}=2^{2} \mathfrak{g}$, since 2 remains prime in $F$ and $(2.8)$ holds. If $m \equiv 2(\bmod 4)$, then $D_{K / F}=2 \mathfrak{g}$, since 2 ramifies in $F$ and we have $(2.8)$. If $m \equiv 3(\bmod 4)$, then $D_{K / F}=\mathfrak{g}$ by $(2.8)$. Let $m \equiv 1(\bmod 8)$. Then 2 splits in $F$. For every $\sigma \in \operatorname{Gal}(K / \mathbb{Q})$, we have $\mathfrak{d}_{K / F}^{\sigma}=\mathfrak{d}_{K / \mathbb{Q}}^{\sigma} /\left(\mathfrak{d}_{F / \mathbb{Q}} \mathfrak{r}\right)^{\sigma}=\mathfrak{d}_{K / \mathbb{Q}} / \mathfrak{d}_{F / \mathbb{Q}} \mathfrak{r}=\mathfrak{d}_{K / F}$, since $K / \mathbb{Q}$ is an abelian extension. Thus $D_{K / F}^{\sigma}=D_{K / F}$, which implies $D_{K / F}=2^{2} \mathfrak{g}$ by $(2.8)$.

Next we prove $(3.5)$. The case $m \equiv 3(\bmod 4)$ is clear. If $m \not \equiv 3(\bmod 4)$, then $v$ is ramified in $K$ for any $v \mid 2$ from (3.4) and Dedekind's discriminant theorem, that is, there exists a prime $w$ in $K$ such that $v \mathfrak{r}=w^{2}$. If $m \equiv 1$ $(\bmod 8)$, then $D_{K / F}=N_{K / F}\left(\mathfrak{d}_{K / F}\right)=2^{2} \mathfrak{g}=\prod_{v} v^{2}$ by (3.4), and hence we have $\mathfrak{d}_{K / F}=\prod_{v \mid 2} \prod_{w \mid v} w^{2}=\prod_{v} v \mathfrak{r}$. The other cases can be shown in the same way.

3.3. The index $\left[\widetilde{L}_{v}: L_{v}\right]$. The index $\left[\widetilde{L}_{v}: L_{v}\right]$ for $v \in \mathbf{h}$ is given by $[6,(3.2 .1)]$ :

$$
\left[\widetilde{L}_{v}: L_{v}\right]= \begin{cases}1 & \text { if } t_{v}=0 \\ {\left[\mathfrak{g}_{v}: 2 \mathfrak{g}_{v}\right]} & \text { if } t_{v}=1 \text { and } v \mid 2, \\ 1 & \text { if } t_{v}=1 \text { and } v \nmid 2, \\ q_{v}^{2} & \text { if } t_{v}=2, \mathfrak{d}_{v}=\mathfrak{r}_{v}, \text { and } c_{v} \in \pi_{v} \mathfrak{g}_{v}^{\times} \\ 1 & \text { if } t_{v}=2, \mathfrak{d}_{v}=\mathfrak{r}_{v}, \text { and } c_{v} \in \mathfrak{g}_{v}^{\times} \\ {\left[\mathfrak{r}_{v}: \mathfrak{d}_{v}\right]} & \text { if } t_{v}=2 \text { and } \mathfrak{d}_{v} \neq \mathfrak{r}_{v}, \\ {\left[\mathfrak{g}_{v}: 2 \mathfrak{g}_{v}\right] q_{v}^{2}} & \text { if } t_{v}=3 \\ q_{v}^{2} & \text { if } t_{v}=4 .\end{cases}
$$

Here $\mathfrak{r}_{v}, \mathfrak{d}_{v}$, and $c_{v}$ are as in $(2.1)$, and $\widetilde{L}_{v}=\left\{y \in\left(F_{v}\right)_{n}^{1} \mid 2 \varphi(x, y) \in \mathfrak{g}_{v}\right.$ for every $\left.x \in L_{v}\right\}$. Using this result, we have the following

Lemma 3.4. Let $L$ be a $\mathfrak{g}$-maximal lattice in $V$ with respect to $\varphi$. Let $v \in \mathbf{h}$.

(1) Suppose $v \nmid 2$. Then $\left[\widetilde{L}_{v}: L_{v}\right]=1$.

(2) Suppose $v \mid 2$.

(i) If $n \equiv 0(\bmod 8)$, then $\left[\widetilde{L}_{v}: L_{v}\right]=1$.

(ii) If $n \equiv \pm 1(\bmod 8)$, then

$$
\left[\widetilde{L}_{v}: L_{v}\right]= \begin{cases}2 & \text { if } m \equiv 1(\bmod 8), \\ 2^{2} & \text { otherwise }\end{cases}
$$


(iii) If $n \equiv \pm 2(\bmod 8)$, then

$$
\left[\widetilde{L}_{v}: L_{v}\right]= \begin{cases}1 & \text { if } m \equiv 3(\bmod 4), \\ 2^{4} & \text { if } m \equiv-3(\bmod 8), \\ 2^{2} & \text { otherwise }\end{cases}
$$

(iv) If $n \equiv \pm 3(\bmod 8)$, then

$$
\left[\widetilde{L}_{v}: L_{v}\right]= \begin{cases}2^{3} & \text { if } m \equiv 1(\bmod 8), \\ 2^{2} & \text { otherwise. }\end{cases}
$$

(v) If $n \equiv 4(\bmod 8)$, then

$$
\left[\widetilde{L}_{v}: L_{v}\right]= \begin{cases}2^{2} & \text { if } m \equiv 1(\bmod 8) \\ 1 & \text { otherwise }\end{cases}
$$

Proof. (1) There exists a $\mathfrak{g}$-maximal lattice $M$ in $V$ with respect to $\varphi$ including $\mathfrak{g}_{n}^{1}$. Then $\mathfrak{g}_{n}^{1} \subset M \subset \widetilde{M} \subset \widetilde{\mathfrak{g}_{n}^{1}}$. Since $\widetilde{\mathfrak{g}_{n}^{1}}=2^{-1} \mathfrak{g}_{n}^{1}$, we have $\widetilde{\left(\mathfrak{g}_{v}\right)_{n}^{1}}=\left(\mathfrak{g}_{v}\right)_{n}^{1}$ for every $v \nmid 2$, so that $\widetilde{M}_{v}=M_{v}$. Since $M$ is in the genus of $L$, there exists $\alpha \in G_{\mathbf{A}}$ such that $L \alpha=M$. Thus we have $L_{v} \alpha_{v}=M_{v}$. Hence $\left[\widetilde{L}_{v}: L_{v}\right]=\left[\widetilde{M}_{v}: M_{v}\right]=1$ if $v \nmid 2$.

(2) Suppose $v \mid 2$. (i) If $n \equiv 0(\bmod 8)$, then $1_{n}$ is equivalent to $\eta_{n / 2}$ over $F_{v}$, that is, $t_{v}=0$ for every $v \in \mathbf{h}$ by Lemma 3.3. Thus the assertion (i) can be easily obtained by (3.6).

(ii) If $n \equiv \pm 1(\bmod 8)$, then $t_{v}=1$ for every $v \in \mathbf{h}$ by Lemma 3.3. Thus, by (3.6), we have

$$
\begin{aligned}
{\left[\widetilde{L}_{v}: L_{v}\right] } & =\left[\mathfrak{g}_{v}: 2 \mathfrak{g}_{v}\right] \\
& = \begin{cases}{[\mathfrak{g}: v]} & \text { if } 2 \text { splits in } F, \\
{[\mathfrak{g}: 2 \mathfrak{g}]} & \text { if } 2 \text { remains prime in } F \\
{[\mathfrak{g}: v]^{2}} & \text { if } 2 \text { ramifies in } F\end{cases} \\
& = \begin{cases}2 & \text { if } m \equiv 1(\bmod 8), \\
2^{2} & \text { otherwise }\end{cases}
\end{aligned}
$$

Thus we have (ii).

(iii) If $m \equiv-1(\bmod 8)$, then $t_{v}=0$ by Lemma 3.3 , and so $\left[\widetilde{L}_{v}: L_{v}\right]=1$. If $m \neq \equiv-1(\bmod 8)$, then $t_{v}=2$ by Lemma 3.3. In this case, by Dedekind's discriminant theorem and $(3.4), F_{v}(\sqrt{-1}) / F_{v}$ is a ramified (resp. an unramified) quadratic extension if $m \equiv 1,2(\bmod 4)(\operatorname{resp} . m \equiv 3(\bmod 8))$. Let $m \equiv 3(\bmod 8)$. Then $t_{v}=2$ and $\mathfrak{d}_{v}=\mathfrak{r}_{v}$. Since we can take $\theta_{v}= \pm 1_{2}$ in Lemma 3.3, we have $c_{v}=b_{v}^{-1}= \pm 1 \in \mathfrak{g}_{v}^{\times}$as shown below (2.1). Thus $\left[\widetilde{L}_{v}: L_{v}\right]=1$ by $(3.6)$. If $m \equiv 1,2(\bmod 4)$, then $\mathfrak{d}_{v}=\pi_{K_{v}}^{2} \mathfrak{r}_{v}$ by $(3.5)$. Here $\pi_{K_{v}}$ is a prime element of $K_{v}=F_{v}(\sqrt{-1})$. Thus by (3.6), we have $\left[\begin{array}{lll}\widetilde{L}_{v} & : L_{v}\end{array}\right]=\left[\begin{array}{lll}\mathfrak{r}_{v} & : & \mathfrak{d}_{v}\end{array}\right]=\left[\begin{array}{lll}\mathfrak{r}_{v} & : \pi_{K_{v}}^{2} \mathfrak{r}_{v}\end{array}\right]$. Since $v$ ramifies in $K$, we have 
$\mathfrak{r}_{v} / \pi_{K_{v}}^{2} \mathfrak{r}_{v} \cong \mathfrak{g}_{v} / \pi_{v} \mathfrak{g}_{v}$, and hence $\left[\mathfrak{r}_{v}: \pi_{K_{v}}^{2} \mathfrak{r}_{v}\right]=q_{v}^{2}$. Therefore

$$
\left[\widetilde{L}_{v}: L_{v}\right]=q_{v}^{2}= \begin{cases}2^{2} & \text { if } m \equiv 1, \pm 2(\bmod 8) \\ 2^{4} & \text { if } m \equiv-3(\bmod 8)\end{cases}
$$

(iv) Let $n \equiv \pm 3(\bmod 8)$. Combining Lemma 3.3 with $(3.6)$, we have

$$
\left[\widetilde{L}_{v}: L_{v}\right]= \begin{cases}{\left[\mathfrak{g}_{v}: 2 \mathfrak{g}_{v}\right] q_{v}^{2}} & \text { if } m \equiv 1(\bmod 8), \\ {\left[\mathfrak{g}_{v}: 2 \mathfrak{g}_{v}\right]} & \text { if } m \neq \equiv 1(\bmod 8),\end{cases}
$$

and hence we get (iv).

(v) Let $n \equiv 4(\bmod 8)$. Then

$$
\left[\widetilde{L}_{v}: L_{v}\right]= \begin{cases}q_{v}^{2} & \text { if } m \equiv 1(\bmod 8) \\ 1 & \text { if } m \neq 1(\bmod 8)\end{cases}
$$

by Lemma 3.3 and (3.6), which proves (v).

We note that $\left[\widetilde{L}_{v}: L_{v}\right] \neq 1$ if and only if $v \mid D_{K / F}$ when $n \equiv \pm 2(\bmod 8)$.

It is well known that $[\widetilde{L}: L]=\prod_{v \in \mathbf{h}}\left[\widetilde{L}_{v}: L_{v}\right]$. Combining this with Lemma 3.4, we have the following

Lemma 3.5. Let $L$ be a $\mathfrak{g}$-maximal lattice in $V$ with respect to $\varphi$.

(1) If $n \equiv 0(\bmod 8)$, then $[\widetilde{L}: L]=1$.

(2) If $n \equiv \pm 1(\bmod 8)$, then $[\widetilde{L}: L]=2^{2}$.

(3) If $n \equiv \pm 2(\bmod 8)$, then

$$
[\widetilde{L}: L]= \begin{cases}1 & \text { if } m \equiv 3(\bmod 4) \\ 2^{4} & \text { if } m \equiv-3(\bmod 8) \\ 2^{2} & \text { otherwise. }\end{cases}
$$

(4) If $n \equiv \pm 3(\bmod 8)$, then

$$
[\widetilde{L}: L]= \begin{cases}2^{6} & \text { if } m \equiv 1(\bmod 8), \\ 2^{2} & \text { otherwise. }\end{cases}
$$

(5) If $n \equiv 4(\bmod 8)$, then

$$
[\widetilde{L}: L]= \begin{cases}2^{4} & \text { if } m \equiv 1(\bmod 8), \\ 1 & \text { otherwise. }\end{cases}
$$

3.4. Formula for computation. Summing up Lemmas 3.3-3.5 and Proposition 2.1, we obtain the following

THEOREM 3.6. Let $F=\mathbb{Q}(\sqrt{m})$ with a square free positive integer $m$, and let $n>1$. Let $L$ be a $\mathfrak{g}$-maximal lattice in $V$ with respect to $\varphi$. Let $\chi, \chi^{\prime}$, and $\chi^{\prime \prime}$ be the Dirichlet characters corresponding to $F / \mathbb{Q}, \mathbb{Q}(\sqrt{-1}) / \mathbb{Q}$, and $\mathbb{Q}(\sqrt{-m}) / \mathbb{Q}$, respectively. 
(1) If $n \equiv 0(\bmod 8)$, then

$$
\mathfrak{m}(L)=n^{-2} B_{n / 2} B_{n / 2, \chi}\left(\prod_{k=1}^{[(n-1) / 2]}(4 k)^{-2} B_{2 k} B_{2 k, \chi}\right) .
$$

(2) If $n \equiv \pm 1(\bmod 8)$, then

$$
\mathfrak{m}(L)=\prod_{k=1}^{(n-1) / 2}(4 k)^{-2} B_{2 k} B_{2 k, \chi} .
$$

(3) If $n \equiv \pm 2(\bmod 8)$, then

$$
\begin{aligned}
\mathfrak{m}(L)= & n^{-2} B_{n / 2, \chi^{\prime}} B_{n / 2, \chi^{\prime \prime}}\left(\prod_{k=1}^{[(n-1) / 2]}(4 k)^{-2} B_{2 k} B_{2 k, \chi}\right) \\
& \times \begin{cases}2^{-2} & \text { if } m \equiv 1(\bmod 8), \\
1 & \text { if } m \equiv 3(\bmod 4), \\
2^{-1} & \text { otherwise. }\end{cases}
\end{aligned}
$$

(4) If $n \equiv \pm 3(\bmod 8)$, then

$$
\begin{aligned}
\mathfrak{m}(L)= & \left(\prod_{k=1}^{(n-1) / 2}(4 k)^{-2} B_{2 k} B_{2 k, \chi}\right) \\
& \times \begin{cases}2^{-2} \cdot 3^{-2}\left(2^{n-1}-1\right)^{2} & \text { if } m \equiv 1(\bmod 8), \\
1 & \text { otherwise. }\end{cases}
\end{aligned}
$$

(5) If $n \equiv 4(\bmod 8)$, then

$$
\begin{aligned}
\mathfrak{m}(L)= & n^{-2} B_{n / 2} B_{n / 2, \chi}\left(\prod_{k=1}^{[(n-1) / 2]}(4 k)^{-2} B_{2 k} B_{2 k, \chi}\right) \\
& \times \begin{cases}2^{-2} \cdot 3^{-2}\left(2^{n / 2-1}-1\right)^{2}\left(2^{n / 2}-1\right)^{2} & \text { if } m \equiv 1(\bmod 8), \\
1 & \text { otherwise. }\end{cases}
\end{aligned}
$$

Proof. (1) If $n \equiv 0(\bmod 8)$, then substituting $\mathfrak{e}=\mathfrak{g}$ and $[\widetilde{L}: L]=1$ into (2.3), we obtain (1).

(2) If $n \equiv \pm 1(\bmod 8)$, then $t_{v}=1$ for every $v \in \mathbf{h}$ and $\mathfrak{f}=\mathfrak{g}$ by Lemma 3.3. By Lemma 3.4, we have $\mathfrak{e}=\prod_{v \mid 2} v$. Hence by Lemma 3.5 and (2.1),

$$
[\widetilde{L}: L]^{(n-1) / 2} \prod_{v \mid \mathfrak{e}} \lambda_{v}=2^{n-1} .
$$

Substituting this into (2.2), we obtain (2).

(3) Suppose $n \equiv \pm 2(\bmod 8)$. If $m \equiv 1,2(\bmod 4)$ and $v \mid 2$, then $t_{v}=2$ by Lemma 3.3. By (2.1) and Lemma 3.4, we have $\lambda_{v}=2^{-1}$ and $\mathfrak{e}=\prod_{v \mid 2} v$. 
If $m \equiv 3(\bmod 4)$, then $\mathfrak{e}=\mathfrak{g}$. Thus by Lemma 3.5 ,

$$
[\widetilde{L}: L]^{(n-1) / 2} \prod_{v \mid \mathfrak{e}} \lambda_{v}= \begin{cases}2^{2(n-2)} & \text { if } m \equiv 1(\bmod 8), \\ 2^{2 n-3} & \text { if } m \neq \equiv-3(\bmod 8), \\ 2^{n-2} & \text { if } m \equiv 2(\bmod 4), \\ 1 & \text { if } m \equiv 3(\bmod 4) .\end{cases}
$$

Combining this with (2.4), we obtain (3).

(4) Let $m \equiv 1(\bmod 8)$. By Lemma 3.3 , we have $t_{v}=3$ if $v \mid 2$. Thus, by Lemma $3.4, \mathfrak{e}=\prod_{v \mid 2} v=\mathfrak{f}$. If $m \not \equiv 1(\bmod 8)$, then $\mathfrak{f}=\mathfrak{g}$ and $\mathfrak{e}=\prod_{v \mid 2} v$. Hence by Lemma 3.5,

$$
2^{1-n}[\widetilde{L}: L]^{(n-1) / 2} \prod_{v \mid \mathfrak{e}} \lambda_{v}= \begin{cases}2^{-2} \cdot 3^{-2}\left(2^{n-1}-1\right)^{2} & \text { if } m \equiv 1(\bmod 8), \\ 1 & \text { otherwise. }\end{cases}
$$

(5) Suppose $n \equiv 4(\bmod 8)$. If $m \equiv 1(\bmod 8)$, then $t_{v}=4$ for $v \mid 2$ and $\mathfrak{e}=\prod_{v \mid 2} v$ by Lemmas 3.3 and 3.4. If $m \not \equiv 1(\bmod 8)$, then $\mathfrak{e}=\mathfrak{g}$. By Lemma 3.5,

$$
[\widetilde{L}: L]^{(n-1) / 2} \prod_{v \mid \mathfrak{e}} \lambda_{v}= \begin{cases}2^{-2} \cdot 3^{-2}\left(2^{n / 2-1}-1\right)^{2}\left(2^{n / 2}-1\right)^{2} & \text { if } m \equiv 1(\bmod 8), \\ 1 & \text { otherwise }\end{cases}
$$

which proves (5).

4. Numerical example. In this section, we take $F=\mathbb{Q}(\sqrt{5})$ and $\varphi=1_{4}$, and as an application of Theorem 3.6 we show that the genus of a $\mathfrak{g}$-maximal lattice in $V=F_{4}^{1}$ with respect to $\varphi$ consists of one class; moreover, we give a formula for $N(L, h)$ by using this fact and the formula due to Shimura in [7, Theorem 1.5].

Let $\mathfrak{g}=\mathbb{Z}+\mathbb{Z} \omega$ with $\omega=(1+\sqrt{5}) / 2$. We note that $\omega$ is a fundamental unit of $F$. We consider a $\mathfrak{g}$-maximal lattice $L$ with respect to $\varphi$. Set

$$
L=\sum_{i=1}^{4} \mathfrak{g} \alpha_{i}=\mathfrak{g}_{4}^{1} \alpha, \quad \alpha=\left[\begin{array}{c}
\alpha_{1} \\
\vdots \\
\alpha_{4}
\end{array}\right] \in \mathrm{GL}_{4}(F) .
$$

A $\mathfrak{g}$-lattice $L$ in $V$ is $\mathfrak{g}$-maximal with respect to $\varphi$ if and only if $\varphi[x] \in \mathfrak{g}$ for every $x \in L$ and $\left[\widetilde{L}_{v}: L_{v}\right]=1$ for every $v \in \mathbf{h}$. These conditions are equivalent to

$$
\left\{\begin{array}{l}
\varphi\left[\alpha_{i}\right] \in \mathfrak{g} \quad(1 \leq i \leq 4), \quad 2 \varphi\left(\alpha_{i}, \alpha_{j}\right) \in \mathfrak{g} \quad(i \neq j) \\
\operatorname{det}(\alpha) \in 2^{-2} \mathfrak{g}^{\times}
\end{array}\right.
$$


The last condition can be seen by using elementary divisors. Put

$$
\alpha=\left[\begin{array}{cccc}
1 & 0 & 0 & 0 \\
0 & 1 & 0 & 0 \\
1 / 2 & (1+\omega) / 2 & \omega / 2 & 0 \\
1 / 2 & 1 / 2 & 1 / 2 & 1 / 2
\end{array}\right]
$$

then $\alpha$ satisfies (4.1). Hence $L=\mathfrak{g}_{4}^{1} \alpha$ is a $\mathfrak{g}$-maximal lattice with respect to $\varphi$. We remark that $L$ contains $\mathfrak{g}_{4}^{1}$.

We shall calculate the order of $\Gamma=\{\gamma \in G \mid L \gamma=L\}$ for this $L$. We take the subgroup

$$
\Gamma_{1}=\left\{\gamma \in \Gamma \mid e_{1} \gamma=e_{1}\right\}
$$

where $\left\{e_{i}\right\}_{i=1}^{4}$ is the standard basis of $V$. We will calculate $\left[\Gamma: \Gamma_{1}\right]$ and $\left[\Gamma_{1}: 1\right]$. For $\gamma={ }^{t}\left[{ }^{t} \gamma_{1} \ldots{ }^{t} \gamma_{4}\right] \in F_{4}^{4}, \gamma \in \Gamma_{1}$ if and only if

$$
\left\{\begin{array}{l}
\gamma_{i} \in n(L, 1) \quad(1 \leq i \leq 4), \quad \gamma_{i} \cdot{ }^{t} \gamma_{j}=0 \quad(i \neq j), \\
2^{-1}\left(\gamma_{1}+(1+\omega) \gamma_{2}+\omega \gamma_{3}\right) \in n(L, 1+\omega), \\
2^{-1}\left(\gamma_{1}+\gamma_{2}+\gamma_{3}+\gamma_{4}\right) \in n(L, 1) .
\end{array}\right.
$$

Here $n(M, h)=\{x \in M \mid \varphi[x]=h\}$ for a $\mathfrak{g}$-lattice $M$ in $V$ and $h \in F$. This can be verified as in [2].

We need $n(L, 1)$ to use condition (4.3). As $\mathfrak{g}_{4}^{1} \subset L$, we see that $L \subset \widetilde{L}$ $\subset \widetilde{\mathfrak{g}_{4}^{1}}=\left(2^{-1} \mathfrak{g}\right)_{4}^{1}$. So we only have to determine $n\left(\left(2^{-1} \mathfrak{g}\right)_{4}^{1}, 1\right)$, and find the elements in the set satisfying the following condition: For $y=\left[x_{1} / 2 \cdots x_{4} / 2\right] \epsilon$ $\left(2^{-1} \mathfrak{g}\right)_{4}^{1}$ and $x_{i}=a_{i}+b_{i} \omega$, the condition $y \in L$ is equivalent to the conditions

$$
\left\{\begin{array}{l}
a_{2}+b_{3}+a_{4}+b_{4} \equiv b_{2}+a_{3}+b_{3}+a_{4} \equiv 0(\bmod 2), \\
a_{1}+a_{3}+b_{3}+b_{4} \equiv b_{1}+a_{3}+a_{4}+b_{4} \equiv 0(\bmod 2) .
\end{array}\right.
$$

The set $n\left(\left(2^{-1} \mathfrak{g}\right)_{4}^{1}, 1\right)$ can be determined in the following way: Let $x / 2$ be an element of $\left(2^{-1} \mathfrak{g}\right)_{4}^{1}$, and solve the equation $\varphi[x]=4$ instead of $\varphi[x / 2]=1$. Furthermore, $\varphi[x]$ is of the form

$$
\varphi[x]=\left[\begin{array}{cc}
1_{4} & 0 \\
0 & 1_{4}
\end{array}\right][\widehat{x}]+\left[\begin{array}{cc}
0 & 1_{4} \\
1_{4} & 1_{4}
\end{array}\right][\widehat{x}] \cdot \omega .
$$

Here we set $\widehat{x}=\left[\begin{array}{llllll}a_{1} & \cdots & a_{4} & b_{1} & \cdots & b_{4}\end{array}\right] \in \mathbb{Z}_{8}^{1}$ for $x=\left[\begin{array}{llll}x_{1} & \cdots & x_{4}\end{array}\right] \in \mathfrak{g}_{4}^{1}$ and $x_{i}=a_{i}+b_{i} \omega$. Calculating $\widehat{x}$ in $\mathbb{Z}_{8}^{1}$ satisfying the conditions

$$
\left\{\begin{array}{l}
{\left[\begin{array}{cc}
1_{4} & 0 \\
0 & 1_{4}
\end{array}\right][\widehat{x}]=4} \\
{\left[\begin{array}{cc}
0 & 1_{4} \\
1_{4} & 1_{4}
\end{array}\right][\widehat{x}]=0}
\end{array}\right.
$$


we get $n\left(\left(2^{-1} \mathfrak{g}\right)_{4}^{1}, 1\right)$. Employing condition (4.4) for this set, we find

$$
\begin{aligned}
n(L, 1)= & n\left(\left(2^{-1} \mathfrak{g}\right)_{4}^{1}, 1\right) \cap L \\
= & \left\{ \pm e_{i} \mid 1 \leq i \leq 4\right\} \cup\left\{\left(\delta_{1} / 2, \ldots, \delta_{4} / 2\right)\right\} \\
& \cup\left\{\left(0, \delta_{2} / 2, \delta_{3} \omega / 2, \delta_{4} \omega^{\varrho} / 2\right)\right\} \cup\left\{\left(0, \delta_{2} \omega / 2, \delta_{3} \omega^{\varrho} / 2, \delta_{4} / 2\right)\right\} \\
& \cup\left\{\left(0, \delta_{2} \omega^{\varrho} / 2, \delta_{3} / 2, \delta_{4} \omega / 2\right)\right\} \cup\left\{\left(\delta_{1} / 2,0, \delta_{3} \omega^{\varrho} / 2, \delta_{4} \omega / 2\right)\right\} \\
& \cup\left\{\left(\delta_{1} \omega / 2,0, \delta_{3} / 2, \delta_{4} \omega^{\varrho} / 2\right)\right\} \cup\left\{\left(\delta_{1} \omega^{\varrho} / 2,0, \delta_{3} \omega / 2, \delta_{4} / 2\right)\right\} \\
& \cup\left\{\left(\delta_{1} / 2, \delta_{2} \omega / 2,0, \delta_{4} \omega^{\varrho} / 2\right)\right\} \cup\left\{\left(\delta_{1} \omega / 2, \delta_{2} \omega^{\varrho} / 2,0, \delta_{4} / 2\right)\right\} \\
& \cup\left\{\left(\delta_{1} \omega^{\varrho} / 2, \delta_{2} / 2,0, \delta_{4} \omega / 2\right)\right\} \cup\left\{\left(\delta_{1} / 2, \delta_{2} \omega^{\varrho} / 2, \delta_{3} \omega / 2,0\right)\right\} \\
& \cup\left\{\left(\delta_{1} \omega / 2, \delta_{2} / 2, \delta_{3} \omega^{\varrho} / 2,0\right)\right\} \cup\left\{\left(\delta_{1} \omega^{\varrho} / 2, \delta_{2} \omega / 2, \delta_{3} / 2,0\right)\right\} .
\end{aligned}
$$

Here we allow $\delta_{i}$ to take the values \pm 1 at random in each set, and $\omega^{\varrho}=$ $1-\omega=-\omega^{-1}$ with the nontrivial automorphism $\varrho$ of $F$ over $\mathbb{Q}$.

We compute $\left[\Gamma: \Gamma_{1}\right]$. For $\gamma, \delta \in \Gamma$, we denote by $\gamma_{1}$ the first row vector of $\gamma \in \Gamma$. Since $\Gamma_{1} \gamma=\Gamma_{1} \delta$ if and only if $\gamma_{1}=\delta_{1}$, we see that $\left[\Gamma: \Gamma_{1}\right] \leq N(L, 1)$; moreover, for any $\gamma_{1} \in n(L, 1)$ there exists an element of $\Gamma$ which has $\gamma_{1}$ as the first row vector. This can be verified by using $n(L, 1)$ and taking an element of $\Gamma$ satisfying (4.3). Thus

$$
\left[\Gamma: \Gamma_{1}\right]=N(L, 1)=120 .
$$

To compute $\left[\Gamma_{1}: 1\right]$, we use the condition

$$
2^{-1}\left(\omega^{-1} \gamma_{1}+\omega^{-1}(1+\omega) \gamma_{2}+\gamma_{3}\right) \in n(L, 1)
$$

instead of

$$
2^{-1}\left(\gamma_{1}+(1+\omega) \gamma_{2}+\omega \gamma_{3}\right) \in n(L, 1+\omega)
$$

in $(4.3)$, because $n(L, 1+\omega)=\omega \cdot n(L, 1)$. From this, $\Gamma_{1}$ consists of all elements $\gamma={ }^{t}\left[{ }^{t} e_{1}{ }^{t} \gamma_{2}{ }^{t} \gamma_{3}{ }^{t} \gamma_{4}\right] \in F_{4}^{4}$ such that

$$
\left\{\begin{array}{l}
\gamma_{i} \in n(L, 1) \quad(2 \leq i \leq 4), \quad \gamma_{i} \cdot{ }^{t} \gamma_{j}=0 \quad(i \neq j) \\
2^{-1}\left(\omega^{-1} \gamma_{1}+\omega^{-1}(1+\omega) \gamma_{2}+\gamma_{3}\right) \in n(L, 1) \\
2^{-1}\left(\gamma_{1}+\gamma_{2}+\gamma_{3}+\gamma_{4}\right) \in n(L, 1)
\end{array}\right.
$$

Since $n(L, 1)$ has been determined, we can choose the pair $\left(e_{1}, \gamma_{2}, \gamma_{3}, \gamma_{4}\right)$ satisfying (4.5). After some calculations, we obtain $\left[\Gamma_{1}: 1\right]=120$. Thus $[\Gamma: 1]=120 \cdot 120=2^{6} \cdot 3^{2} \cdot 5^{2}$.

On the other hand, we see that $\mathfrak{m}(L)=2^{-6} \cdot 3^{-2} \cdot 5^{-2}$ by Theorem 3.6. Therefore the genus of $L$ consists of one class. 
Next, to introduce the formula in [7, Theorem 1.5(II)], we define $\gamma_{v}(s)$ by the following formulas (cf. $[7, \S 1.6])$ :

$$
\gamma_{v}(s)= \begin{cases}\frac{1-q_{v}^{(\nu+1)(1-s)}}{1-q_{v}^{1-s}} & \text { if } t_{v}=0, \\ \frac{1-\left(-q_{v}^{1-s}\right)^{\nu+1}}{1+q_{v}^{1-s}} & \text { if } t_{v}=2, \mathfrak{d}_{v}=\mathfrak{r}_{v}, \text { and } c_{v} \in \mathfrak{g}_{v}^{\times}, \\ \frac{1-\left(-q_{v}^{1-s}\right)^{\nu+2}+q_{v}^{2-s}\left(1-\left(-q_{v}^{1-s}\right)^{\nu}\right)}{\left(1+q_{v}^{-s}\right)\left(1+q_{v}^{1-s}\right)} & \text { if } t_{v}=2, \mathfrak{d}_{v}=\mathfrak{r}_{v}, \text { and } c_{v} \in \pi_{v} \mathfrak{g}_{v}^{\times}, \\ 1+\psi_{v}\left(c_{v} h\right)\left(|h|_{v}^{-1} N\left(\mathfrak{d}_{v}\right)\right)^{1-s} & \text { if } t_{v}=2 \text { and } \mathfrak{d}_{v} \neq \mathfrak{r}_{v}, \\ \frac{1-q_{v}^{(\nu+2)(1-s)}-q_{v}^{2-s}\left(1-q_{v}^{\nu(1-s)}\right)}{\left(1-q_{v}^{-s}\right)\left(1-q_{v}^{1-s}\right)} & \text { if } t_{v}=4,\end{cases}
$$

where $\nu \in \mathbb{Z}$ is determined by $|h|_{v}^{-1}=q_{v}^{\nu}$ with the normalized valuation ||$_{v}$ at $v$ of $F_{v}$.

THEOREM 4.1 (Shimura). Let $F$ be a totally real algebraic number field, and put $n=2 u$ and $V=F_{n}^{1}$. Let $\varphi$ be a totally positive symmetric matrix of $\mathrm{GL}_{n}(F), L$ a $\mathfrak{g}$-maximal lattice in $V$ with respect to $\varphi$, and $h$ a totally positive element of $\mathfrak{g}$. Put $K=F\left(\sqrt{(-1)^{n / 2} \operatorname{det}(\varphi)}\right)$, and denote by $\mathfrak{d}$ the different of $K / F$ and by $\psi_{K / F}$ the Hecke character of $F$ corresponding to the extension $K / F$. Then

$$
\begin{aligned}
\frac{R(L, h)}{\mathfrak{m}(L)}= & \frac{N_{F / \mathbb{Q}}(h)^{u-1}}{D_{F}^{(n-1) / 2} N(\mathfrak{d})^{1 / 2} N(\mathfrak{f})\left\{(u-1) !(2 \pi)^{-u}\right\}^{2} L\left(u, \psi_{K / F}\right)} \\
& \times \prod_{v \mid h \mathfrak{e}} \gamma_{v}(u) .
\end{aligned}
$$

Here $\mathfrak{e}$ is the product of all primes $v$ satisfying $\widetilde{L}_{v} \neq L_{v}, \mathfrak{f}$ is the product of prime factors of $\mathfrak{e}$ unramified in $K$, and $\gamma_{v}(s)$ is given as above.

We specialize this formula to the case where $F=\mathbb{Q}(\sqrt{5}), V=F_{4}^{1}$, and $\varphi=1_{4}$. We easily see that $K=F$. By Lemma 3.4 , we have $\mathfrak{e}=\mathfrak{g}$. Since $t_{v}=0$ for every $v \in \mathbf{h}$ by Lemma $3.3, \gamma_{v}(2)$ is given by

$$
\gamma_{v}(2)=\frac{1-q_{v}^{-\left(e_{v}+1\right)}}{1-q_{v}^{-1}}
$$

for a totally positive element $h$ of $\mathfrak{g}$, where the principal ideal of $h$ is 
$(h)_{F}=\prod_{v} v^{e_{v}}$. Now, the genus of a $\mathfrak{g}$-maximal lattice with respect to $\varphi$ consists of one class. Thus $N(L, h)=R(L, h) \mathfrak{m}(L)^{-1}$. Hence by Theorem 4.1, we obtain

$$
\begin{aligned}
N(L, h) & =\frac{N_{F / \mathbb{Q}}(h)}{5^{3 / 2}(2 \pi)^{-4} \zeta_{F}(2)} \prod_{v \mid h} \frac{1-q_{v}^{-\left(e_{v}+1\right)}}{1-q_{v}^{-1}} \\
& =120 \prod_{v \mid h}\left(\sum_{i=0}^{e_{v}} q_{v}^{i}\right) .
\end{aligned}
$$

Thus $N(L, h) / 120$ coincides with the Fourier coefficient of the Eisenstein series $E_{2}$ in the space $\mathcal{M}_{(2,2)}(\mathfrak{g}$, id) of all Hilbert modular forms for GL(2) over $F=\mathbb{Q}(\sqrt{5})$ of weight $(2,2)$, level $\mathfrak{g}$, and with the identity character. It is known that the dimension of this space is 1 (see [3, §3.4], for example), that is, $\mathcal{M}_{(2,2)}(\mathfrak{g}, \mathrm{id})=\mathbb{C} \cdot E_{2}$ in this case.

5. Numerical table of $\mathfrak{m}(L)$. Let $F=\mathbb{Q}(\sqrt{m})$ with a square free positive integer $m$, and let $\varphi=1_{n}$. Using Theorem 3.6, we give a table of $\mathfrak{m}(L)$ for several quadratic fields $F$.

We note that the $l$ th generalized Bernoulli number $B_{l, \psi}$ associated with a Dirichlet character $\psi$ can be computed by the following recursion formula (cf. [4]):

$$
\begin{aligned}
B_{l, \psi} & =\frac{1}{f} \sum_{a=1}^{f} \psi(a) a^{l}-\sum_{k=1}^{l-1} \frac{f^{l-k}}{k}\left(\begin{array}{c}
l \\
k-1
\end{array}\right) B_{k, \psi} \quad(0<l \in \mathbb{Z}), \\
B_{0, \psi} & =0 .
\end{aligned}
$$

\begin{tabular}{|c|c|c|c|c|c|}
\hline$m n$ & 2 & 3 & 4 & 5 & 6 \\
\hline 2 & $\frac{1}{2^{4}}$ & $\frac{1}{2^{4} \cdot 3}$ & $\frac{1}{2^{8} \cdot 3^{2}}$ & $\frac{11}{2^{9} \cdot 3^{2} \cdot 5}$ & $\frac{11}{2^{13 \cdot 3 \cdot 5}}$ \\
\hline 3 & $\frac{1}{2^{3} \cdot 3}$ & $\frac{1}{2^{3} \cdot 3}$ & $\frac{1}{2^{6} \cdot 3^{2}}$ & $\frac{23}{2^{7} \cdot 3^{2} \cdot 5}$ & $\frac{23}{2^{9} \cdot 3^{4} \cdot 5}$ \\
\hline 5 & $\frac{1}{2^{3}}$ & $\frac{1}{2^{3} \cdot 3 \cdot 5}$ & $\frac{1}{2^{6} \cdot 3^{2} \cdot 5^{2}}$ & $\frac{1}{2^{7} \cdot 3^{2} \cdot 5^{2}}$ & $\frac{1}{2^{10} \cdot 3 \cdot 5}$ \\
\hline 6 & $\frac{1}{2^{3}}$ & $\frac{1}{2^{3}}$ & $\frac{1}{2^{6}}$ & $\frac{3 \cdot 29}{2^{7} \cdot 5}$ & $\frac{3 \cdot 23 \cdot 29}{2^{10} \cdot 5}$ \\
\hline 7 & $\frac{1}{2^{3}}$ & $\frac{1}{2 \cdot 3}$ & $\frac{1}{2^{2} \cdot 3^{2}}$ & $\frac{113}{2^{3} \cdot 3^{2} \cdot 5}$ & $\frac{113}{2^{2} \cdot 3^{2} \cdot 5 \cdot 7}$ \\
\hline 10 & $\frac{1}{2^{3}}$ & $\frac{7}{2^{3} \cdot 3}$ & $\frac{7^{2}}{2^{6} \cdot 3^{2}}$ & $\frac{7 \cdot 19 \cdot 83}{2^{7} \cdot 3^{2} \cdot 5}$ & $\frac{7 \cdot 19 \cdot 79 \cdot 83}{2^{10} \cdot 3^{2} \cdot 5}$ \\
\hline 11 & $\frac{1}{2^{3}}$ & $\frac{7}{2^{3} \cdot 3}$ & $\frac{7^{2}}{2^{6} \cdot 3^{2}}$ & $\frac{7 \cdot 2153}{2^{7} \cdot 3^{2} \cdot 5}$ & $\frac{7 \cdot 2153}{2^{9} \cdot 3 \cdot 5}$ \\
\hline 13 & $\frac{1}{2^{3}}$ & $\frac{1}{2^{3} \cdot 3}$ & $\frac{1}{2^{6} \cdot 3^{2}}$ & $\frac{29}{2^{7} \cdot 3^{2} \cdot 5}$ & $\frac{29 \cdot 151}{2^{10} \cdot 3^{2} \cdot 5}$ \\
\hline 14 & $\frac{1}{2^{2}}$ & $\frac{5}{2^{2 \cdot 3}}$ & $\frac{5^{2}}{2^{4} \cdot 3^{2}}$ & $\frac{2503}{2^{5} \cdot 3^{2}}$ & $\frac{11 \cdot 2503}{2^{7}}$ \\
\hline
\end{tabular}

Here $f$ is the conductor of $\psi$ and we put $\left(\begin{array}{l}l \\ r\end{array}\right)=l ! /(l-r) ! r !$. 


\begin{tabular}{|c|c|c|c|c|c|}
\hline$m n$ & 2 & 3 & 4 & 5 & 6 \\
\hline 15 & $\frac{1}{2^{2}}$ & $\frac{1}{2}$ & $\frac{1}{2^{2}}$ & $\frac{3 \cdot 179}{2^{3} \cdot 5}$ & $\frac{3 \cdot 179}{2^{2} \cdot 5}$ \\
\hline 17 & $\frac{1}{2^{3}}$ & $\frac{1}{2^{4} \cdot 3}$ & $\frac{1}{2^{6} \cdot 3^{2}}$ & $\frac{5 \cdot 41}{2^{7} \cdot 3^{2}}$ & $\frac{41 \cdot 53}{2^{8} \cdot 3 \cdot 5}$ \\
\hline 19 & $\frac{1}{2^{3}}$ & $\frac{19}{2^{3} \cdot 3}$ & $\frac{19^{2}}{2^{6} \cdot 3^{2}}$ & $\frac{19 \cdot 109 \cdot 137}{2^{7} \cdot 3^{2} \cdot 5}$ & $\frac{11 \cdot 19 \cdot 109 \cdot 137}{2^{9} \cdot 3^{2} \cdot 5}$ \\
\hline 21 & $\frac{1}{2^{2}}$ & $\frac{1}{2^{2} \cdot 3}$ & $\frac{1}{2^{4} \cdot 3^{2}}$ & $\frac{7 \cdot 11}{2^{5} \cdot 3^{2} \cdot 5}$ & $\frac{7 \cdot 11 \cdot 263}{2^{7} \cdot 3^{2} \cdot 5}$ \\
\hline 22 & $\frac{1}{2^{3}}$ & $\frac{23}{2^{3} \cdot 3}$ & $\frac{23^{2}}{2^{6} \cdot 3^{2}}$ & $\frac{23 \cdot 24889}{2^{7} \cdot 3^{2} \cdot 5}$ & $\frac{13 \cdot 23 \cdot 43 \cdot 24889}{2^{10} \cdot 3^{2} \cdot 5}$ \\
\hline 23 & $\frac{3}{2^{3}}$ & $\frac{5}{2 \cdot 3}$ & $\frac{5^{2}}{2^{2} \cdot 3^{2}}$ & $\frac{41 \cdot 173}{2^{3} \cdot 3^{2}}$ & $\frac{41 \cdot 173}{2^{2} \cdot 3}$ \\
\hline 26 & $\frac{3}{2^{3}}$ & $\frac{5^{2}}{2^{3} \cdot 3}$ & $\frac{5^{4}}{2^{6} \cdot 3^{2}}$ & $\frac{5 \cdot 31 \cdot 1409}{2^{7} \cdot 3^{2}}$ & $\frac{5 \cdot 31 \cdot 311 \cdot 1409}{2^{10} \cdot 3}$ \\
\hline 29 & $\frac{3}{2^{3}}$ & $\frac{1}{2^{3}}$ & $\frac{1}{2^{6}}$ & $\frac{157}{2^{7} \cdot 5}$ & $\frac{3 \cdot 11 \cdot 37 \cdot 157}{2^{10 \cdot 5}}$ \\
\hline 30 & $\frac{1}{2^{2}}$ & $\frac{17}{2^{2} \cdot 3}$ & $\frac{17^{2}}{2^{4} \cdot 3^{2}}$ & $\frac{17 \cdot 36451}{2^{5} \cdot 3^{2} \cdot 5}$ & $\frac{17 \cdot 127 \cdot 36451}{2^{7} \cdot 3^{2}}$ \\
\hline 31 & $\frac{3}{2^{3}}$ & $\frac{5}{3}$ & $\frac{5^{2}}{3^{2}}$ & $\frac{10357}{2 \cdot 3^{2}}$ & $\frac{2 \cdot 10357}{3}$ \\
\hline 33 & $\frac{1}{2^{3}}$ & $\frac{1}{2^{4}}$ & $\frac{1}{2^{6}}$ & $\frac{3 \cdot 5 \cdot 47}{2^{7}}$ & $\frac{3 \cdot 11 \cdot 47 \cdot 73}{2^{8} \cdot 5}$ \\
\hline 34 & $\frac{1}{2^{2}}$ & $\frac{23}{2^{2} \cdot 3}$ & $\frac{23^{2}}{2^{4} \cdot 3^{2}}$ & $\frac{23 \cdot 57241}{2^{5} \cdot 3^{2} \cdot 5}$ & $\frac{7 \cdot 11^{2} \cdot 23 \cdot 57241}{2^{7} \cdot 3^{2} \cdot 5}$ \\
\hline 35 & $\frac{1}{2^{2}}$ & $\frac{19}{2^{2} \cdot 3}$ & $\frac{19^{2}}{2^{4} \cdot 3^{2}}$ & $\frac{7 \cdot 19 \cdot 8819}{2^{5} \cdot 3^{2} \cdot 5}$ & $\frac{3 \cdot 7 \cdot 19 \cdot 8819}{2^{6} \cdot 5}$ \\
\hline 37 & $\frac{1}{2^{3}}$ & $\frac{5}{2^{3} \cdot 3}$ & $\frac{5^{2}}{2^{6} \cdot 3^{2}}$ & $\frac{1129}{2^{7} \cdot 3^{2}}$ & $\frac{23 \cdot 89 \cdot 1129}{2^{10} \cdot 3^{2}}$ \\
\hline 38 & $\frac{3}{2^{3}}$ & $\frac{41}{2^{3} \cdot 3}$ & $\frac{41^{2}}{2^{6} \cdot 3^{2}}$ & $\frac{23 \cdot 41 \cdot 1429}{2^{7} \cdot 3^{2}}$ & $\frac{7 \cdot 23 \cdot 41 \cdot 113 \cdot 1429}{2^{10} \cdot 3}$ \\
\hline 39 & $\frac{1}{2}$ & $\frac{13}{2 \cdot 3}$ & $\frac{13^{2}}{2^{2} \cdot 3^{2}}$ & $\frac{5 \cdot 13 \cdot 31 \cdot 59}{2^{3} \cdot 3^{2}}$ & $\frac{5 \cdot 11 \cdot 13 \cdot 31 \cdot 59}{2^{2} \cdot 3^{2}}$ \\
\hline 41 & $\frac{1}{2^{2}}$ & $\frac{1}{2^{2} \cdot 3}$ & $\frac{1}{2^{2} \cdot 3^{2}}$ & $\frac{2^{2} \cdot 5 \cdot 7}{3^{2}}$ & $\frac{2^{2} \cdot 3^{4} \cdot 7}{5}$ \\
\hline 42 & $\frac{1}{2^{2}}$ & $\frac{3^{2}}{2^{2}}$ & $\frac{3^{4}}{2^{4}}$ & $\frac{3^{4} \cdot 5^{3} \cdot 7}{2^{5}}$ & $\frac{3^{4} \cdot 5^{3} \cdot 7^{2} \cdot 11 \cdot 19}{2^{7}}$ \\
\hline 43 & $\frac{1}{2^{3}}$ & $\frac{3 \cdot 7}{2^{3}}$ & $\frac{3^{2} \cdot 7^{2}}{2^{6}}$ & $\frac{3 \cdot 7 \cdot 11 \cdot 7873}{2^{7} \cdot 5}$ & $\frac{3 \cdot 7 \cdot 11 \cdot 83 \cdot 7873}{2^{9} \cdot 5}$ \\
\hline 46 & $\frac{1}{2^{2}}$ & $\frac{37}{2^{2} \cdot 3}$ & $\frac{37^{2}}{2^{4} \cdot 3^{2}}$ & $\frac{37 \cdot 164999}{2^{5} \cdot 3^{2} \cdot 5}$ & $\frac{37 \cdot 359 \cdot 164999}{2^{7} \cdot 3^{2}}$ \\
\hline 47 & $\frac{5}{2^{3}}$ & $\frac{7}{3}$ & $\frac{7^{2}}{3^{2}}$ & $\frac{7 \cdot 43223}{2 \cdot 3^{2} \cdot 5}$ & $\frac{2 \cdot 7 \cdot 43223}{5}$ \\
\hline 51 & $\frac{1}{2^{2}}$ & $\frac{13}{2^{2}}$ & $\frac{13^{2}}{2^{4}}$ & $\frac{3 \cdot 13 \cdot 5197}{2^{5}}$ & $\frac{3 \cdot 13 \cdot 67 \cdot 5197}{2^{6}}$ \\
\hline 53 & $\frac{3}{2^{3}}$ & $\frac{7}{2^{3} \cdot 3}$ & $\frac{7^{2}}{2^{6} \cdot 3^{2}}$ & $\frac{5^{2} \cdot 7 \cdot 31}{2^{7} \cdot 3^{2}}$ & $\frac{5^{2} \cdot 7 \cdot 13 \cdot 31 \cdot 139}{2^{10} \cdot 3}$ \\
\hline 55 & $\frac{1}{2}$ & $\frac{23}{2 \cdot 3}$ & $\frac{23^{2}}{2^{2} \cdot 3^{2}}$ & $\frac{23^{2} \cdot 6689}{2^{3} \cdot 3^{2} \cdot 5}$ & $\frac{5 \cdot 23^{2} \cdot 6689}{2^{2} \cdot 3^{2}}$ \\
\hline 57 & $\frac{1}{2^{3}}$ & $\frac{7}{2^{4} \cdot 3}$ & $\frac{7^{2}}{2^{6} \cdot 3^{2}}$ & $\frac{5 \cdot 7 \cdot 47 \cdot 61}{2^{7} \cdot 3^{2}}$ & $\frac{7 \cdot 31 \cdot 47 \cdot 61 \cdot 101}{2^{8} \cdot 3^{2} \cdot 5}$ \\
\hline 58 & $\frac{1}{2^{3}}$ & $\frac{3 \cdot 11}{2^{3}}$ & $\frac{3^{2} \cdot 11^{2}}{2^{6}}$ & $\frac{3 \cdot 11 \cdot 246839}{2^{7} \cdot 5}$ & $\frac{3 \cdot 11 \cdot 1259 \cdot 246839}{2^{10}}$ \\
\hline 59 & $\frac{3}{2^{3}}$ & $\frac{5 \cdot 17}{2^{3} \cdot 3}$ & $\frac{5^{2} \cdot 17^{2}}{2^{6} \cdot 3^{2}}$ & $\frac{17 \cdot 271 \cdot 2837}{2^{7} \cdot 3^{2}}$ & $\frac{17 \cdot 67 \cdot 271 \cdot 2837}{2^{9} \cdot 3}$ \\
\hline 61 & $\frac{3}{2^{3}}$ & $\frac{11}{2^{3} \cdot 3}$ & $\frac{11^{2}}{2^{6} \cdot 3^{2}}$ & $\frac{11 \cdot 17 \cdot 383}{2^{7} \cdot 3^{2} \cdot 5}$ & $\frac{3 \cdot 11 \cdot 17 \cdot 271 \cdot 383}{2^{10} \cdot 5}$ \\
\hline 62 & $\frac{1}{2}$ & $\frac{7}{2}$ & $\frac{7^{2}}{2^{2}}$ & $\frac{7 \cdot 15193}{2^{3}}$ & $\frac{3 \cdot 7 \cdot 673 \cdot 15193}{2^{4}}$ \\
\hline 65 & $\frac{1}{2^{2}}$ & $\frac{1}{2 \cdot 3}$ & $\frac{1}{3^{2}}$ & $\frac{5 \cdot 1123}{2^{2} \cdot 3^{2}}$ & $\frac{11 \cdot 23 \cdot 1123}{2^{2} \cdot 5}$ \\
\hline 66 & $\frac{1}{2}$ & $\frac{2 \cdot 7}{3}$ & $\frac{2^{2} \cdot 7^{2}}{3^{2}}$ & $\frac{7 \cdot 29 \cdot 4967}{3^{2} \cdot 5}$ & $\frac{7 \cdot 29 \cdot 2309 \cdot 4967}{2 \cdot 3^{2} \cdot 5}$ \\
\hline
\end{tabular}




\begin{tabular}{|c|c|c|c|c|c|}
\hline$m n$ & 2 & 3 & 4 & 5 & 6 \\
\hline 70 & $\frac{1}{2^{2}}$ & $\frac{67}{2^{2} \cdot 3}$ & $\frac{67^{2}}{2^{4} \cdot 3^{2}}$ & $\frac{5 \cdot 7 \cdot 67 \cdot 4091}{2^{5} \cdot 3^{2}}$ & $\frac{5^{2} \cdot 7 \cdot 67 \cdot 1019 \cdot 4091}{2^{7} \cdot 3^{2}}$ \\
\hline 71 & $\frac{7}{2^{3}}$ & $\frac{29}{2 \cdot 3}$ & $\frac{29^{2}}{2^{2} \cdot 3^{2}}$ & $\frac{11 \cdot 29 \cdot 101 \cdot 331}{2^{3} \cdot 3^{2} \cdot 5}$ & $\frac{11 \cdot 17 \cdot 29 \cdot 101 \cdot 331}{2^{2} \cdot 3 \cdot 5}$ \\
\hline 73 & $\frac{1}{2^{3}}$ & $\frac{11}{2^{4} \cdot 3}$ & $\frac{11^{2}}{2^{6} \cdot 3^{2}}$ & $\frac{5^{2} \cdot 7 \cdot 11 \cdot 197}{2^{7} \cdot 3^{2}}$ & $\frac{5 \cdot 7^{3} \cdot 11 \cdot 23 \cdot 197}{2^{8} \cdot 3^{2}}$ \\
\hline 74 & $\frac{5}{2^{3}}$ & $\frac{41}{2^{3}}$ & $\frac{41^{2}}{2^{6}}$ & $\frac{31 \cdot 41 \cdot 101 \cdot 181}{2^{7} \cdot 5}$ & $\frac{3^{2} \cdot 17 \cdot 31 \cdot 41 \cdot 83 \cdot 101 \cdot 181}{2^{10} \cdot 5}$ \\
\hline 77 & $\frac{1}{2}$ & $\frac{1}{2}$ & $\frac{1}{2^{2}}$ & $\frac{1193}{2^{3} \cdot 5}$ & $\frac{3 \cdot 1153 \cdot 1193}{2^{4} \cdot 5}$ \\
\hline 78 & $\frac{1}{2^{2}}$ & $\frac{23}{2^{2}}$ & $\frac{23^{2}}{2^{4}}$ & $\frac{3 \cdot 23 \cdot 31 \cdot 3697}{2^{5} \cdot 5}$ & $\frac{3 \cdot 13 \cdot 17 \cdot 23 \cdot 31^{2} \cdot 3697}{2^{7} \cdot 5}$ \\
\hline 79 & $\frac{5}{2^{3}}$ & 7 & $7^{2}$ & $\frac{7 \cdot 37 \cdot 2467}{2 \cdot 5}$ & $\frac{2 \cdot 7 \cdot 31 \cdot 37 \cdot 2467}{5}$ \\
\hline 82 & $\frac{1}{2^{2}}$ & $\frac{3^{3}}{2^{2}}$ & $\frac{3^{6}}{2^{4}}$ & $\frac{3^{3} \cdot 179 \cdot 463}{2^{5}}$ & $\frac{3^{3} \cdot 17 \cdot 179 \cdot 443 \cdot 463}{2^{7}}$ \\
\hline 83 & $\frac{3}{2^{3}}$ & $\frac{43}{2^{3}}$ & $\frac{43^{2}}{2^{6}}$ & $\frac{7^{3} \cdot 43 \cdot 2459}{2^{7} \cdot 5}$ & $\frac{3 \cdot 7^{3} \cdot 31 \cdot 43 \cdot 2459}{2^{9}}$ \\
\hline 85 & $\frac{1}{2^{2}}$ & $\frac{3}{2^{2}}$ & $\frac{3^{2}}{2^{4}}$ & $\frac{3 \cdot 3463}{2^{5.5}}$ & $\frac{3 \cdot 3463 \cdot 8263}{2^{7 \cdot 5}}$ \\
\hline 86 & $\frac{5}{2^{3}}$ & $\frac{5 \cdot 31}{2^{3} \cdot 3}$ & $\frac{5^{2} \cdot 31^{2}}{2^{6} \cdot 3^{2}}$ & $\frac{11 \cdot 29^{2} \cdot 31 \cdot 311}{2^{7} \cdot 3^{2}}$ & $\frac{7 \cdot 11 \cdot 29^{2} \cdot 31 \cdot 293 \cdot 311}{2^{10}}$ \\
\hline 87 & $\frac{3}{2^{2}}$ & $\frac{13}{2}$ & $\frac{13^{2}}{2^{2}}$ & $\frac{3 \cdot 13 \cdot 83903}{2^{3} \cdot 5}$ & $\frac{3^{5} \cdot 13 \cdot 83903}{2^{2} \cdot 5}$ \\
\hline 89 & $\frac{3}{2^{3}}$ & $\frac{13}{2^{4} \cdot 3}$ & $\frac{13^{2}}{2^{6} \cdot 3^{2}}$ & $\frac{5^{2} \cdot 13 \cdot 37 \cdot 73}{2^{7} \cdot 3^{2}}$ & $\frac{13 \cdot 37 \cdot 73 \cdot 3371}{2^{8} \cdot 3}$ \\
\hline 91 & $\frac{1}{2^{2}}$ & $\frac{103}{2^{2} \cdot 3}$ & $\frac{103^{2}}{2^{4} \cdot 3^{2}}$ & $\frac{7 \cdot 13 \cdot 103 \cdot 19739}{2^{5} \cdot 3^{2} \cdot 5}$ & $\frac{5 \cdot 7 \cdot 11 \cdot 13 \cdot 103 \cdot 19739}{2^{6} \cdot 3^{2}}$ \\
\hline 93 & $\frac{1}{2^{2}}$ & $\frac{3}{2^{2}}$ & $\frac{3^{2}}{2^{4}}$ & $\frac{3 \cdot 4679}{2^{5} \cdot 5}$ & $\frac{3 \cdot 4679 \cdot 10631}{2^{7} \cdot 5}$ \\
\hline 94 & $\frac{1}{2}$ & $\frac{53}{2 \cdot 3}$ & $\frac{53^{2}}{2^{2} \cdot 3^{2}}$ & $\frac{41 \cdot 53 \cdot 24527}{2^{3} \cdot 3^{2} \cdot 5}$ & $\frac{13 \cdot 41 \cdot 53 \cdot 83 \cdot 24527}{2^{4} \cdot 3^{2}}$ \\
\hline 95 & 1 & $\frac{43}{2 \cdot 3}$ & $\frac{43^{2}}{2^{2} \cdot 3^{2}}$ & $\frac{43 \cdot 1017299}{2^{3} \cdot 3^{2} \cdot 5}$ & $\frac{7 \cdot 43 \cdot 1017299}{2^{2} \cdot 3}$ \\
\hline 97 & $\frac{1}{2^{3}}$ & $\frac{17}{2^{4} \cdot 3}$ & $\frac{17^{2}}{2^{6} \cdot 3^{2}}$ & $\frac{5 \cdot 17^{2} \cdot 1097}{2^{7} \cdot 3^{2}}$ & $\frac{17^{2} \cdot 29 \cdot 79 \cdot 1097}{2^{8} \cdot 3^{2}}$ \\
\hline
\end{tabular}

\section{References}

[1] M. Deuring, Algebren, Zweite, korrigierte auflage, Ergeb. Math. Grenzgeb. 41, Springer, Berlin, 1968.

[2] T. Hiraoka, On the class number of the genus of $\mathbb{Z}$-maximal lattices for quadratic form of the sum of squares, preprint.

[3] H. Ishikawa, The traces of Hecke operators in the space of the 'Hilbert modular' type cusp forms of weight two, Sci. Papers College Gen. Ed. Univ. Tokyo 29 (1979), $1-28$.

[4] K. Iwasawa, Lectures on p-adic L-functions, Ann. of Math. Stud. 74, Princeton Univ. Press, 1972.

[5] G. Shimura, Euler Products and Eisenstein Series, CBMS Reg. Conf. Ser. Math. 93, Amer. Math. Soc., Providence, 1997.

[6] —, An exact mass formula for orthogonal groups, Duke Math. J. 99 (1999), $1-66$.

[7] -, The number of representations of an integer by a quadratic form, ibid. 100 (1999), $59-92$. 
[8] G. Shimura, The representation of integers as sums of squares, Amer. J. Math. 124 (2002), 1059-1081.

Department of Mathematical Sciences

Ritsumeikan University

Kusatsu, Shiga 525-8577, Japan

E-mail: rp056956@se.ritsumei.ac.jp

Received on 20.12.2004

and in revised form on 6.10.2005 